\title{
Impact of shell crossing and scope of perturbative approaches, in real and redshift space
}

\begin{abstract}
P. Valageas
Institut de Physique Théorique, CEA Saclay, 91191 Gif-sur-Yvette, France

e-mail: patrick.valageas@cea.fr

Received 30 August 2010 / Accepted 21 October 2010

ABSTRACT

Aims. We study the effect of nonperturbative corrections associated with the behavior of particles after shell crossing on the matter power spectrum. We compare their amplitude with the perturbative terms that can be obtained within the fluid description of the system, to estimate the range of scales where such perturbative approaches are relevant.

Methods. We use the simple Zeldovich dynamics as a benchmark, as it allows the exact computation of the full nonlinear power spectrum and of perturbative terms at all orders. Then, we introduce a "sticky model" that coincides with the Zeldovich dynamics before shell crossing but shows a different behavior afterwards. Thus, their power spectra only differ in their nonperturbative terms. We consider both the real-space and redshift-space power spectra.

Results. We find that the potential of perturbative schemes is greater at higher redshift for a $\Lambda$ CDM cosmology. For the real-space power spectrum, one can go up to order 66 of perturbation theory at $z=3$, and to order 9 at $z=0$, before the nonperturbative correction surpasses the perturbative correction of that order. This allows us to increase the upper bound on $k$ where systematic theoretical predictions may be obtained by perturbative schemes, beyond the linear regime, by a factor $\sim 26$ at $z=3$ and $\sim 6.5$ at $z=0$. This provides a strong motivation to study perturbative resummation schemes, especially at high redshifts $z \geq 1$. In the context of cosmological reconstruction methods, the Monge-Ampère-Kantorovich scheme appears to be close to optimal at $z=0$. There also seems to be little room for improvement over current reconstruction methods of the baryon acoustic oscillations at $z=0$. This can be understood from the small number of perturbative terms that are relevant at $z=0$, before nonperturbative corrections dominate. We also point out that the rise of the power spectrum on the transition scale to the nonlinear regime strongly depends on the behavior of the system after shell crossing. We find similar results for the redshift-space power spectrum, with characteristic wavenumbers that are shifted to lower values as redshift-space distortions amplify higher order terms of the perturbative expansions while decreasing the resummed nonlinear power at high $k$.
\end{abstract}

Key words. gravitation - large-scale structure of Universe

\section{Introduction}

The growth of large-scale structures in the Universe through the amplification of small primordial fluctuations by gravitational instability is a key ingredient of modern cosmology (Peebles 1980), and it can be used to constrain cosmological parameters through the dependence of the matter power spectrum on scale and redshift. On very large scales or at high redshifts, where the amplitude of the density fluctuations is small, it is sufficient to use linear theory, whereas on small scales, in the highly nonlinear regime, one must use numerical simulations or phenomenological models, such as the halo model (Cooray \& Sheth 2002), which are also calibrated on simulations. In the weakly nonlinear regime one expects perturbative approaches to provide a useful tool, as they allow going beyond linear theory in a systematic and controlled fashion. Several observational probes, such as weak lensing surveys (Massey et al. 2007; Munshi et al. 2008) or measures of acoustic baryonic oscillations (Eisenstein et al. 1998, 2005), are mostly sensitive to these intermediate scales, and to meet the accuracy of future observations we need a theoretical accuracy of about $1 \%$. Phenomenological models typically have an accuracy of $10 \%$ in this range, while numerical simulations may suffer from finite resolution and finite size effects and require a long computational time if we need to obtain the power spectra over a fine grid of cosmological parameters.
This has led to a renewed interest in perturbative approaches, as it may be possible to improve over the standard perturbation theory (Goroff et al. 1986; Bernardeau et al. 2002) by using resummation schemes that allow systematic partial resummations of higher order terms. Thus, Crocce \& Scoccimarro (2006b,a) present a partial resummation of the diagrammatic series associated with the response function (propagator), within a high- $k$ limit, which provides improved predictions for the density power spectrum (Crocce \& Scoccimarro 2008). On the other hand, Valageas (2007a) describes a path-integral formalism that allows applying the tools of field theory, such as large- $N$ expansions, to compute the power spectrum and higher order statistics like the bispectrum (Valageas 2008). One of these large- $N$ expansions was recovered by Taruya \& Hiramatsu (2008), as a "closure theory" where one closes the hierarchy of equations satisfied by the many body correlations at the third order, following the "direct interaction approximation" introduced in hydrodynamics (Kraichnan 1959). This also improves the predictions for the power spectrum on the scales probed by the baryonic acoustic oscillations (Taruya et al. 2009; Valageas \& Nishimichi 2010). Other approaches have been proposed by Matarrese \& Pietroni (2007), using the dependence on a running high- $k$ cutoff, by Pietroni (2008), using a truncation of the hierarchy satisfied by the many-body correlations, by Matsubara (2008), within a Lagrangian framework, and by McDonald (2007), using a 
renormalization group technique. Most $^{1}$ of these approaches start from the fluid description of the system, where the density and velocity fields obey hydrodynamical equations of motion. This corresponds to a single-stream approximation that neglects shell crossing. Then, the domain of validity of most such perturbative schemes is limited to wavenumbers where shellcrossing effects are negligible, even if we could sum all perturbative terms.

This problem with the impact of shell crossing also appears in the context of cosmological reconstruction, where one attempts to follow the matter distribution observed in a given galaxy survey back in time (Peebles 1989). Thus, an efficient algorithm for building such a reconstruction (which can then be used to estimate the velocity field) is provided by the MongeAmpère-Kantorovich method, which neglects multistreaming (Brenier et al. 2003; Mohayaee et al. 2006). Similar methods are used to reconstruct the baryon acoustic oscillations (BAO) of the density power spectrum, in order to improve the accuracy of cosmological distance measurements and tighten the constraints on cosmological parameters such as the amount and evolution of dark energy (Eisenstein et al. 2007; Seo et al. 2010). Then, by comparing the amplitude of nonperturbative corrections with the perturbative terms obtained within the fluid description, one can estimate the scale down to which these reconstruction schemes can be used.

Thus, to estimate the potential of such approaches, it is necessary to evaluate the effect of shell crossing on the matter power spectrum. This question has already been investigated by Afshordi (2007) by comparing the phenomenological halo model with a modified variant where halos are collapsed to pointlike masses. In this paper we revisit this problem in a more systematic fashion, within the framework of the Zeldovich dynamics. Thus, we compare the Zeldovich dynamics with a second model (named the "sticky model" in the following), which only differs after shell crossing. Therefore, both power spectra have the same perturbative expansions and only differ by nonperturbative terms. Taking advantage of our being able to explicitly compute the full nonlinear spectra, as well as perturbative terms at all orders and these nonperturbative corrections, we can compare their respective amplitudes in detail. This allows a more detailed discussion of the importance of shell-crossing effects and of the scope of perturbative approaches. This also enables us to distinguish the dependence on the behavior of the dynamics after shell crossing of the rise of the density power spectrum on mildly nonlinear scales, in the intermediate regime where the logarithmic power goes from $\Delta^{2}(k) \sim 1$ to $\Delta^{2}(k) \sim 100$. In addition, we can perform the same analysis for the redshift-space power spectrum, which is actually the quantity most directly observed in galaxy surveys.

This article is organized as follows. We first recall in Sect. 2.1 the nonlinear real-space power spectrum associated with the Zeldovich dynamics, and its perturbative expansions in Sects. 2.2 and 2.3. We present our "sticky model", which only differs from the Zeldovich dynamics after shell crossing, in Sect. 2.4, and we give the associated nonperturbative correction. Then, we describe the numerical results obtained for a $\Lambda C D M$ cosmology in Sect. 3, comparing the various perturbative and nonperturbative terms. Finally, we extend our analysis to the redshift-space power spectrum in Sect. 4 , focusing on

\footnotetext{
1 Two exceptions are the formalism developed in Valageas (2004), which applies to the Vlasov equation, and the Lagrangian approach of Matsubara (2008), which however is not valid beyond shell crossing.
}

wavenumbers that are aligned with the line of sight. We conclude in Sect. 5.

\section{Computation of the density power spectrum in real space}

\subsection{Nonlinear Zeldovich power spectrum}

As is well known, the Zeldovich approximation (Zeldovich 1970) sets the Eulerian position, $\boldsymbol{x}(\boldsymbol{q}, t)$, of the particle of Lagrangian coordinate $\boldsymbol{q}$, equal to the position given by the linear displacement field, $\boldsymbol{\Psi}_{\mathrm{L}}(\boldsymbol{q}, t)$,

$\boldsymbol{x}(\boldsymbol{q}, t)=\boldsymbol{q}+\boldsymbol{\Psi}_{\mathrm{L}}(\boldsymbol{q}, t)$ with $\nabla_{\boldsymbol{q}} \cdot \boldsymbol{\Psi}_{\mathrm{L}}=-\delta_{\mathrm{L}}(\boldsymbol{q}, t)$,

where $\delta_{\mathrm{L}}(\boldsymbol{q}, t)$ is the linear growing mode of the density contrast, which is defined by

$\delta(\boldsymbol{x}, t)=\frac{\rho(\boldsymbol{x}, t)-\bar{\rho}}{\bar{\rho}}$.

Here $\bar{\rho}$ is the mean matter density of the Universe, we work in comoving coordinates and as usual we only consider the linear growing mode. It is well known (Schneider \& Bartelmann 1995; Taylor \& Hamilton 1996) that the explicit expression of the matter power spectrum can be derived from Eq. (1) by using the conservation of matter, which reads as

$\rho(\boldsymbol{x}) \mathrm{d} \boldsymbol{x}=\bar{\rho} \mathrm{d} \boldsymbol{q} \quad$ whence $1+\delta(\boldsymbol{x})=\left|\operatorname{det}\left(\frac{\partial \boldsymbol{x}}{\partial \boldsymbol{q}}\right)\right|^{-1}$.

For an arbitrary displacement field $\boldsymbol{\Psi}$, this also reads as

$1+\delta(\boldsymbol{x})=\int \mathrm{d} \boldsymbol{q} \delta_{\mathrm{D}}[\boldsymbol{x}-\boldsymbol{q}-\boldsymbol{\Psi}(\boldsymbol{q})]$,

where $\delta_{\mathrm{D}}$ is the Dirac distribution, and this yields in Fourier space (for $k \neq 0$, that is, disregarding a term $\delta_{\mathrm{D}}(\boldsymbol{k})$ ):

$\tilde{\delta}(\boldsymbol{k})=\int \frac{\mathrm{d} \boldsymbol{x}}{(2 \pi)^{3}} \mathrm{e}^{-\mathrm{i} \boldsymbol{k} \cdot \boldsymbol{x}} \delta(\boldsymbol{x})=\int \frac{\mathrm{d} \boldsymbol{q}}{(2 \pi)^{3}} \mathrm{e}^{-\mathrm{i} \boldsymbol{k} \cdot(\boldsymbol{q}+\boldsymbol{\Psi})}$.

Defining the density power spectrum as

$\left\langle\tilde{\delta}\left(\boldsymbol{k}_{1}\right) \tilde{\delta}\left(\boldsymbol{k}_{2}\right)\right\rangle=\delta_{\mathrm{D}}\left(\boldsymbol{k}_{1}+\boldsymbol{k}_{2}\right) P\left(k_{1}\right)$

we obtain from Eq. (5), using statistical homogeneity,

$P(k)=\int \frac{\mathrm{d} \boldsymbol{q}}{(2 \pi)^{3}}\left\langle\mathrm{e}^{\mathrm{i} k \cdot[\boldsymbol{x}(\boldsymbol{q})-x(0)]}\right\rangle$.

Equation (7) is quite general since we have not used the Zeldovich approximation (1) yet. It shows how the density power spectrum is related to the statistical properties of the displacement field, for any mapping $\boldsymbol{q} \mapsto \boldsymbol{x}$ (which can include some shell-crossing, as in the Zeldovich case). The great simplification provided by the Zeldovich approximation (1) is that in this case the quantity $\boldsymbol{x}(\boldsymbol{q})-\boldsymbol{x}(0)$ is a Gaussian random variable, so that the mean can be computed at once as

$P(k)=\int \frac{\mathrm{d} \boldsymbol{q}}{(2 \pi)^{3}} \mathrm{e}^{\mathrm{i} \boldsymbol{k} \cdot \boldsymbol{q}} \mathrm{e}^{-\frac{1}{2}\left\langle\left(\boldsymbol{k} \cdot\left[\boldsymbol{\Psi}_{\mathrm{L}}(\boldsymbol{q})-\boldsymbol{\Psi}_{\mathrm{L}}(0)\right]\right)^{2}\right\rangle}$.

Next, using the second relation (1) to compute the average $\left\langle\left(\boldsymbol{k} \cdot\left[\boldsymbol{\Psi}_{\mathrm{L}}(\boldsymbol{q})-\boldsymbol{\Psi}_{\mathrm{L}}(0)\right]\right)^{2}\right\rangle$, we obtain the explicit expression

$P(k)=\int \frac{\mathrm{d} \boldsymbol{q}}{(2 \pi)^{3}} \mathrm{e}^{\mathrm{i} \boldsymbol{k} \cdot \boldsymbol{q}} \mathrm{e}^{-\int \mathrm{d} \boldsymbol{w}[1-\cos (\boldsymbol{w} \cdot \boldsymbol{q})] \frac{(\boldsymbol{k} \cdot \boldsymbol{w})^{2}}{w^{4}} P_{\mathrm{L}}(w)}$. 
It is convenient to perform the integration over the angles of the wavenumber $\boldsymbol{w}$ by expanding $\mathrm{e}^{\mathrm{i} w \cdot \boldsymbol{q}}$ over spherical harmonics (Schneider \& Bartelmann 1995),

$$
\int \mathrm{d} \boldsymbol{w} \mathrm{e}^{\mathrm{i} w \cdot \boldsymbol{q}} \frac{(\boldsymbol{k} \cdot \boldsymbol{w})^{2}}{w^{4}} P_{\mathrm{L}}(w)=k^{2} I_{0}(q)+k^{2}\left(1-3 \mu^{2}\right) I_{2}(q),
$$

where $\mu=(\boldsymbol{k} \cdot \boldsymbol{q}) /(k q)$, and we introduced

$I_{\ell}(q)=\frac{4 \pi}{3} \int_{0}^{\infty} \mathrm{d} w P_{\mathrm{L}}(w) j_{\ell}(q w)$,

where $j_{\ell}$ is the spherical Bessel function of order $\ell$. In particular, the variance $\sigma_{v}^{2}$ of the one-dimensional displacement field (or of the linear velocity field, up to a time-dependent multiplicative factor), reads as

$\sigma_{v}^{2}=\frac{1}{3}\left\langle\left|\boldsymbol{\Psi}_{\mathrm{L}}\right|^{2}\right\rangle=I_{0}(0)$.

Therefore, the expression (9) also writes as

$P(k)=\int \frac{\mathrm{d} \boldsymbol{q}}{(2 \pi)^{3}} \cos (k q \mu) \mathrm{e}^{-k^{2}\left[\sigma_{v}^{2}-I_{0}(q)-\left(1-3 \mu^{2}\right) I_{2}(q)\right]}$.

Following Schneider \& Bartelmann (1995), we can perform the integration over the angles of $\boldsymbol{q}$ by expanding part of the exponential, and using the property

$$
\int_{0}^{1} \mathrm{~d} \mu \cos (k q \mu)\left(1-\mu^{2}\right)^{\ell}=\ell !\left(\frac{2}{k q}\right)^{\ell} j_{\ell}(k q),
$$

which gives

$P(k)=\int \frac{\mathrm{d} q q^{2}}{2 \pi^{2}} \mathrm{e}^{-k^{2}\left[\sigma_{v}^{2}-I_{0}(q)+2 I_{2}(q)\right]} \sum_{\ell=0}^{\infty}\left(\frac{6 k I_{2}(q)}{q}\right)^{\ell} j_{\ell}(k q)$.

\subsection{Standard perturbative expansion}

In the standard perturbative approach (Goroff et al. 1986; Bernardeau et al. 2002), one writes the nonlinear density contrast as a series over powers of the linear growing mode,

$\tilde{\delta}(\boldsymbol{k})=\sum_{n=1}^{\infty} \tilde{\delta}^{(n)}(\boldsymbol{k}) \quad$ with $\quad \tilde{\delta}^{(n)}(k) \propto\left(\tilde{\delta}_{\mathrm{L}}\right)^{n} ;$

that is,

$$
\begin{aligned}
\tilde{\delta}^{(n)}(\boldsymbol{k})= & \int \mathrm{d} \boldsymbol{w}_{1} . . \mathrm{d} \boldsymbol{w}_{n} \delta_{\mathrm{D}}\left(\boldsymbol{w}_{1}+. .+\boldsymbol{w}_{n}-\boldsymbol{k}\right) F_{n}\left(\boldsymbol{w}_{1}, . ., \boldsymbol{w}_{n}\right) \\
& \times \tilde{\delta}_{\mathrm{L}}\left(\boldsymbol{w}_{1}\right) . . \tilde{\delta}_{\mathrm{L}}\left(\boldsymbol{w}_{n}\right) .
\end{aligned}
$$

Substituting this expansion (and the one associated with the velocity field) into the equations of motion one obtains a recursion relation for the kernels $F_{n}$, which allows terms of increasing order to be computed in a sequential manner. As is well known, for the Zeldovich dynamics, where $\boldsymbol{\Psi}=\boldsymbol{\Psi}_{\mathrm{L}}$, we do need to follow this route, since by expanding the exponential (5) over $\boldsymbol{\Psi}_{\mathrm{L}}$, and using $\tilde{\boldsymbol{\Psi}}_{\mathrm{L}}(\boldsymbol{k})=\mathrm{i}\left(\boldsymbol{k} / k^{2}\right) \tilde{\delta}_{\mathrm{L}}(\boldsymbol{k})$, we obtain at once all terms,

$F_{n}\left(\boldsymbol{w}_{1}, . ., \boldsymbol{w}_{n}\right)=\frac{1}{n !} \frac{\boldsymbol{k} \cdot \boldsymbol{w}_{1}}{w_{1}^{2}} \ldots \frac{\boldsymbol{k} \cdot \boldsymbol{w}_{n}}{w_{n}^{2}}$

Then, substituting the expansion (16) into the definition (6) of the power spectrum and taking the Gaussian average, one obtains the standard perturbative series

$P(k)=\sum_{n=1}^{\infty} P^{(n)}(k)$ with $P^{(n)}(k) \propto\left(P_{\mathrm{L}}\right)^{n}$.
In particular, the two lowest order terms are

$P^{(1)}(k)=P_{\mathrm{L}}(k), \quad P^{(2)}(k)=P_{22}(k)+P_{13}(k)$,

where $P_{22}$ and $P_{13}$ arise from terms of the form $\left\langle\tilde{\delta}^{(2)} \tilde{\delta}^{(2)}\right\rangle$ and $\left\langle\tilde{\delta}^{(1)} \tilde{\delta}^{(3)}\right\rangle$, with

$$
\begin{aligned}
P_{22}(k)= & \int \mathrm{d} \boldsymbol{w}_{1} \mathrm{~d} \boldsymbol{w}_{2} \delta_{\mathrm{D}}\left(\boldsymbol{w}_{1}+\boldsymbol{w}_{2}-\boldsymbol{k}\right) \frac{\left(\boldsymbol{k} \cdot \boldsymbol{w}_{1}\right)^{2}\left(\boldsymbol{k} \cdot \boldsymbol{w}_{2}\right)^{2}}{2 w_{1}^{4} w_{2}^{4}} \\
& \times P_{\mathrm{L}}\left(w_{1}\right) P_{\mathrm{L}}\left(w_{2}\right),
\end{aligned}
$$

and

$P_{13}(k)=-k^{2} \sigma_{v}^{2} P_{\mathrm{L}}(k)$.

In fact, for the Zeldovich dynamics it is not necessary to use this procedure, since by expanding the exponential in the exact result (9) or (15) we obtain all terms at once,

$$
\begin{aligned}
P^{(n)}(k)= & \int_{0}^{\infty} \frac{\mathrm{d} q q^{2}}{2 \pi^{2}} \sum_{p=0}^{n} \frac{1}{p !}\left[k^{2}\left(I_{0}(q)-2 I_{2}(q)-\sigma_{v}^{2}\right)\right]^{p} \\
& \times\left(\frac{6 k I_{2}(q)}{q}\right)^{n-p} j_{n-p}(k q) .
\end{aligned}
$$

\subsection{Renormalized perturbative expansion}

As pointed out by Crocce \& Scoccimarro (2006b), it is useful to keep the $\boldsymbol{q}$-independent exponential term $\mathrm{e}^{-k^{2} \sigma_{v}^{2}}$ in Eq. (13) and to define a "renormalized" perturbative expansion

$P(k)=\mathrm{e}^{-k^{2} \sigma_{v}^{2}} \sum_{n=1}^{\infty} P_{\sigma_{v}}^{(n)}(k)$ with $P_{\sigma_{v}}^{(n)}(k) \propto\left(P_{\mathrm{L}}\right)^{n}$.

Indeed, while the standard perturbative terms $P^{(n)}$ grow increasingly fast at high $k$ and show large cancellations, the "renormalized" terms $\mathrm{e}^{-k^{2} \sigma_{v}^{2}} P_{\sigma_{v}}^{(n)}$ are positive and show a Gaussian decay at high $k$. Thus, each term peaks on a well-defined range of $k$ and one can clearly see the contribution of diagrams of a given order to the full nonlinear power spectrum. In fact, as noticed in Crocce \& Scoccimarro (2006b), these terms also write as

$$
\begin{aligned}
P_{\sigma_{v}}^{(n)}(k)= & n ! \int \mathrm{d} \boldsymbol{w}_{1} . . \mathrm{d} \boldsymbol{w}_{n} \delta_{\mathrm{D}}\left(\boldsymbol{w}_{1}+. .+\boldsymbol{w}_{n}-\boldsymbol{k}\right) \\
& \times F_{n}\left(\boldsymbol{w}_{1}, . ., \boldsymbol{w}_{n}\right)^{2} P_{\mathrm{L}}\left(w_{1}\right) . . P_{\mathrm{L}}\left(w_{n}\right) .
\end{aligned}
$$

This can be seen at once by expanding the expression (9) and recognizing the square of the kernel $F_{n}$ given in Eq. (18). Thus, each new term $P_{\sigma_{v}}^{(n)}$ is associated with the kernel $F_{n}$ that couples $n$ linear modes, so that one can follow the contribution of higher order mode couplings. Again, from Eq. (15) we obtain all terms at once,

$$
\begin{aligned}
P_{\sigma_{v}}^{(n)}(k)= & \int_{0}^{\infty} \frac{\mathrm{d} q q^{2}}{2 \pi^{2}} \sum_{p=0}^{n} \frac{1}{p !}\left[k^{2}\left(I_{0}(q)-2 I_{2}(q)\right)\right]^{p} \\
& \times\left(\frac{6 k I_{2}(q)}{q}\right)^{n-p} j_{n-p}(k q),
\end{aligned}
$$

while the two lowest order terms are

$P_{\sigma_{v}}^{(1)}(k)=P_{\mathrm{L}}(k), \quad P_{\sigma_{v}}^{(2)}(k)=P_{22}(k)$,

where $P_{22}$ was given in Eq. (21). 
For numerical purposes, it is convenient to obtain the standard perturbative terms (19) from the renormalized ones (24). Expanding the prefactor in Eq. (24) gives

$$
P^{(n)}(k)=\sum_{p=0}^{n-1} \frac{1}{p !}\left(-k^{2} \sigma_{v}^{2}\right)^{p} P_{\sigma_{v}}^{(n-p)}(k) .
$$

On the other hand, to compute the full nonlinear power spectrum (15) it is convenient to subtract the two terms $\mathrm{e}^{-k^{2} \sigma_{v}^{2}}\left[P_{\sigma_{v}}^{(1)}(k)+\right.$ $P_{\sigma_{v}}^{(2)}(k)$ ], so as to avoid integrating over badly behaving terms for $q \rightarrow \infty$.

\subsection{Nonperturbative correction}

The expressions recalled in the previous sections give the nonlinear power spectrum and its perturbative expansions for the usual Zeldovich dynamics, where particles follow the linear trajectories (1). This includes shell crossing and leads to a decay of the nonlinear power spectrum at high $k$, as particles freely escape to infinity, and these random trajectories erase small-scale features (Schneider \& Bartelmann 1995; Taylor \& Hamilton 1996; Valageas 2007b; Bernardeau \& Valageas 2010a). However, because of the simple nature of the Zeldovich dynamics, the full nonlinear result (13) can be obtained by resumming ${ }^{2}$ the perturbative expansions (19) or (24). The latter can be obtained from the usual hydrodynamical equations of motion in Eulerian space, which actually break down at shell crossing.

Our goal in this work is to compare the perturbative terms (19) and (24) with the nonperturbative terms that are associated with the physics that takes place after shell crossing. Within the framework of the Zeldovich dynamics considered in this article, this means that we wish to compare the previous results with those that would be obtained for a second dynamics, which coincides with the Zeldovich dynamics until shell crossing. Then, the difference between both power spectra would give us the amplitude of the correction due to shell-crossing effects, which are generically nonperturbative.

To introduce this second model, let us first recall that the linear growing mode of the displacement field, $\boldsymbol{\Psi}_{\mathrm{L}}(\boldsymbol{q})$, and the associated peculiar velocity field, $\boldsymbol{v}_{\mathrm{L}}(\boldsymbol{q}) \propto \boldsymbol{\Psi}_{\mathrm{L}}(\boldsymbol{q})$, are curlfree (Peebles 1980), and are derived from a velocity potential, $\boldsymbol{v}_{\mathrm{L}}=$ $-\nabla \cdot \chi_{\mathrm{L}}$. Using the Poisson equation we can see that this potential is equal to the linear gravitational potential $\Phi_{\mathrm{L}}$, up to a timedependent factor (Peebles 1980; Vergassola et al. 1994). In particular, the Lagrangian-space to Eulerian-space mapping defined by the Zeldovich dynamics (1) derives from a Lagrangian potential $\varphi_{\mathrm{L}}(\boldsymbol{q})$,

$\boldsymbol{x}(\boldsymbol{q}, t)=\frac{\partial \varphi_{\mathrm{L}}}{\partial \boldsymbol{q}}$

with

$\varphi_{\mathrm{L}}(\boldsymbol{q}, t)=\frac{|\boldsymbol{q}|^{2}}{2}-\frac{a(t)}{4 \pi \mathcal{G} \bar{\rho}} \Phi_{\mathrm{L}}(\boldsymbol{q}, t)$,

where $\Phi_{\mathrm{L}}$ is the linear gravitational potential, which obeys the Poisson equation,

$\Delta_{q} \Phi_{\mathrm{L}}=\frac{4 \pi \mathcal{G} \bar{\rho}}{a(t)} \delta_{\mathrm{L}}$

\footnotetext{
2 The radius of convergence of such series can be finite (e.g., for a power-law linear spectrum $P_{\mathrm{L}}(k) \propto k^{n}$ with $n=-2$ ) or zero (if $n<-2$ ), see Valageas (2007b).
}

Here $\mathcal{G}$ is Newton's constant and $a(t)$ the scale factor. Then, from Eq. (3) the nonlinear density contrast is given by the Hessian determinant of $\varphi_{\mathrm{L}}$,

$$
1+\delta(\boldsymbol{x})=\left|\operatorname{det}\left(\frac{\partial \boldsymbol{x}}{\partial \boldsymbol{q}}\right)\right|^{-1}=\left|\operatorname{det}\left(\frac{\partial^{2} \varphi_{\mathrm{L}}}{\partial q_{i} \partial q_{j}}\right)\right|^{-1} .
$$

At early times, $t \rightarrow 0$, (and if there is not too much power on small scales), the Lagrangian potential $\varphi_{\mathrm{L}}$ is dominated by the first term in Eq. (30) and $\boldsymbol{x}(\boldsymbol{q}, t) \rightarrow \boldsymbol{q}$. Then, the Hessian matrix $\left(\partial^{2} \varphi_{\mathrm{L}} / \partial q_{i} \partial q_{j}\right)$ is definite positive and goes to the identity matrix, whence $\delta \rightarrow 0$. As time increases and structures form, the Lagrangian potential becomes increasingly sensitive to the fluctuations in the linear gravitational potential, and the Hessian determinant deviates from unity. However, the eigenvalues remain strictly positive until shell crossing, which means that the Lagrangian potential $\varphi_{\mathrm{L}}$ remains a strictly convex function. Then, at shell crossing one eigenvalue goes through zero and becomes negative (generically the collapse proceeds at different rates along the three axes). Thus, as is known (Vergassola et al. 1994; Brenier et al. 2003; Bernardeau \& Valageas 2010b), the onset of shell crossing is associated with the change in sign of the Hessian determinant of $\varphi_{\mathrm{L}}$ and with the loss of convexity of the Lagrangian potential $\varphi_{\mathrm{L}}$ (the Hessian matrix is no longer positive-definite).

A well-studied dynamics that agrees with the Zeldovich dynamics until shell crossing is provided by the "adhesion model" (Gurbatov et al. 1989, 1991; Vergassola et al. 1994). More precisely, the "geometrical adhesion model" (Bernardeau \& Valageas 2010b; Valageas \& Bernardeau 2010) leads to replacing the linear Lagrangian potential $\varphi_{\mathrm{L}}(\boldsymbol{q})$, which defines the mapping $\boldsymbol{q} \mapsto \boldsymbol{x}$ through (29), by a nonlinear Lagrangian potential $\varphi$ given by the convex hull of $\varphi_{\mathrm{L}}$, that is, $\varphi=\operatorname{conv}\left(\varphi_{\mathrm{L}}\right)$. Then, particles no longer cross each other but form shocks. Moreover, this second dynamics still coincides with the Zeldovich dynamics outside of shocks, where $\varphi_{\mathrm{L}}$ coincides with its convex hull.

In this article, we do not compute the density power spectrum associated with this "adhesion model", or another explicit dynamical system, which is a difficult task. Since we are merely interested in the amplitude of the effects associated with shell crossing, we simply make use of the property that shell crossing is associated with the loss of convexity of the Lagrangian potential $\varphi_{\mathrm{L}}(\boldsymbol{q})$. Then, we note that as long as $\varphi_{\mathrm{L}}(\boldsymbol{q})$ is strictly convex its restriction along a line that goes through two arbitrary points $\boldsymbol{q}_{A}$ and $\boldsymbol{q}_{B}$ is also strictly convex, which implies (see also Noullez \& Vergassola 1994; Brenier et al. 2003),

before shell crossing, for any $\boldsymbol{q}_{A} \neq \boldsymbol{q}_{B}$ :

$$
\left[\boldsymbol{x}\left(\boldsymbol{q}_{B}\right)-\boldsymbol{x}\left(\boldsymbol{q}_{A}\right)\right] \cdot\left(\boldsymbol{q}_{B}-\boldsymbol{q}_{A}\right)>0 .
$$

Indeed, we can choose a coordinate system so that $\boldsymbol{q}_{A}=0$ and $\boldsymbol{q}_{B}=(q, 0,0)$, and from strict convexity we obtain $\partial^{2} \varphi_{\mathrm{L}} / \partial q_{1}^{2}>0$, whence $\partial x_{1} / \partial q_{1}>0$ along the first axis and $x_{1}(q)-x_{1}(0)>0$, which reads as (33) in its general form ${ }^{3}$. This means that the projection of the Eulerian separation vector, $\Delta x=x_{B}-x_{A}$, onto

3 In 1D the property (33) is an obvious consequence of the absence of shell crossing, but this is not the case in higher dimensions. Indeed, the result (33) makes use of the constraints associated with the fact that the Lagrangian mapping derives from a Lagrangian potential as in (29). This relies on the Zeldovich dynamics and on the linear growing mode of the velocity and displacement fields being curlfree. For the gravitational dynamics, there is no such clear signature of the absence of shell crossing, since even in regular regions the displacement field $\Psi(\boldsymbol{q}, t)$ develops rotational terms at the third order of perturbation theory (Buchert 1994; Bernardeau \& Valageas 2008). 
the Lagrangian vector, $\Delta \boldsymbol{q}=\boldsymbol{q}_{B}-\boldsymbol{q}_{A}$, is positive, so that $\Delta \boldsymbol{x}$ lies in the forward half-space delimited by the plane orthogonal to $\Delta \boldsymbol{q}$.

Going back to the general expression (7), the density power spectrum is fully determined by the mean $\left\langle\mathrm{e}^{\mathrm{i} k \cdot \Delta x}\right\rangle_{\boldsymbol{q}}$, for any $\boldsymbol{q}$, where we note $\Delta \boldsymbol{x}=\boldsymbol{x}(\boldsymbol{q})-\boldsymbol{x}(0)$. To compute this quantity we can take $\boldsymbol{q}$ along the first axis, that is, $\boldsymbol{q}=q \boldsymbol{e}_{1}$ with $q=|\boldsymbol{q}|$. Then, before shell crossing we have from the constraint (33) the property $\Delta x_{1}>0$. Therefore, we consider the "sticky model" defined by:

"sticky model", for $\boldsymbol{q}=|\boldsymbol{q}| \boldsymbol{e}_{1}: \Delta x_{1}=\max \left(\Delta x_{L 1}, 0\right)$,

where $\Delta \boldsymbol{x}_{\mathrm{L}}$ is the linear Eulerian separation, as given by Eq. (1). Thus, this second model only differs from the Zeldovich dynamics when the parallel linear Eulerian separation, $\Delta x_{L 1}$, is negative, in which case we set it equal to zero. This is thus a simplified version of the "adhesion model", as once $\Delta x_{L 1}$ reaches zero, it remains equal to zero forever. However, the model (34) cannot be explicitly derived from the "adhesion model", since we take neither transverse directions nor larger scales into account. Therefore, we use the more generic name "sticky model", to refer to this sticking along one direction for the pair separation.

It is clear that the condition $\Delta x_{L 1}<0$, where the two models differ, is only a sufficient condition for shell crossing, but it is not necessary. Thus, it is a local condition that does not take the "cloud-in-cloud" problem into account: even though no shell crossing seems to have appeared on scale $q$ yet, it may happen that this region is enclosed within a larger domain of size $L$ that has already collapsed, so that particles in the smaller domain have actually experienced shell crossing (Bond et al. 1991). In terms of the Lagrangian potential $\varphi_{\mathrm{L}}$, which defines the Lagrangian mapping, $\boldsymbol{q} \mapsto \boldsymbol{x}$, through Eq. (29), the absence of shell crossing on a small domain of size $q$ means that $\varphi_{\mathrm{L}}$ is equal to its convex hull in this domain (Vergassola et al. 1994; Bec \& Khanin 2007; Bernardeau \& Valageas 2010b). However, the construction of the convex hull is a global problem, as one must consider the behavior of $\varphi_{\mathrm{L}}(\boldsymbol{q})$ over all the space, thereby taking into account the "cloud-in-cloud" problem, while in the definition of the model (34) we only check a weaker condition, since we only consider the two points 0 and $\boldsymbol{q}$. This means that we somewhat underestimate the effects of shell crossing, but we can expect to obtain a reasonable estimate of their amplitude because the probability of collapse decreases on larger scales and we perform a statistical integration over the angles of $\boldsymbol{q}$ in Eq. (7).

From the previous discussions, the "sticky model" (34) and the Zeldovich dynamics (1) coincide before shell crossing, since then we have $\Delta x_{L 1}>0$. This implies that both theories coincide at all orders of the perturbation theory; that is, they show the same expansions (23) and (26) over powers of $P_{\mathrm{L}}$. However, they differ through nonperturbative terms, which arise from their different behaviors after shell crossing.

From Eq. (34) we obtain $\Delta \Psi_{1}=\max \left(\Delta \Psi_{L 1},-q\right)$, for $\boldsymbol{q}=$ $q \boldsymbol{e}_{1}$, and

$$
\begin{aligned}
\left\langle\mathrm{e}^{\mathrm{i} k \cdot \Delta x}\right\rangle_{q \boldsymbol{e}_{1}}= & \mathrm{e}^{\mathrm{i} \boldsymbol{k} \cdot \boldsymbol{q}} \mathrm{e}^{-\int \mathrm{d} \boldsymbol{w}[1-\cos (\boldsymbol{w} \cdot \boldsymbol{q})] \frac{(\boldsymbol{k} \cdot \boldsymbol{w})^{2}}{w^{4}} P_{\mathrm{L}}(w)} \\
& \times \mathrm{e}^{\frac{1}{2} k_{1}^{2} \sigma_{\|}^{2}} \int_{-\infty}^{\infty} \frac{\mathrm{d} \Delta \Psi_{L 1}}{\sqrt{2 \pi} \sigma_{\|}} \mathrm{e}^{-\left(\Delta \Psi_{L 1}\right)^{2} /\left(2 \sigma_{\|}^{2}\right)} \mathrm{e}^{\mathrm{i} k_{1} \Delta \Psi_{1}},
\end{aligned}
$$

where we factorized the result associated with the usual Zeldovich dynamics (8) in the first two terms and we introduced the variance of the linear longitudinal displacement,

$$
\begin{aligned}
\sigma_{\|}^{2}(q) & =\left\langle\left(\Delta \Psi_{L 1}\right)^{2}\right\rangle=2 \int \mathrm{d} w\left[1-\cos \left(w_{1} q\right)\right] \frac{w_{1}^{2}}{w^{4}} P_{\mathrm{L}}(w) \\
& =2 \sigma_{v}^{2}-2 I_{0}(q)+4 I_{2}(q) .
\end{aligned}
$$

Separating the contribution from $\Delta \Psi_{L 1}<-q$, we obtain for the last two terms of Eq. (35),

$$
\begin{aligned}
& \mathrm{e}^{\frac{1}{2} k_{1}^{2} \sigma_{\|}^{2}\left\langle\mathrm{e}^{\mathrm{i} k_{1} \Delta \Psi_{1}}\right\rangle=} 1+\mathrm{e}^{\frac{1}{2} k_{1}^{2} \sigma_{\|}^{2}} \int_{-\infty}^{-q} \frac{\mathrm{d} \Delta \Psi_{L 1}}{\sqrt{2 \pi} \sigma_{\|}} \mathrm{e}^{-\left(\Delta \Psi_{L 1}\right)^{2} /\left(2 \sigma_{\|}^{2}\right)} \\
& \times\left(\mathrm{e}^{-\mathrm{i} k_{1} q}-\mathrm{e}^{\mathrm{i} k_{1} \Delta \Psi_{L 1}}\right) \\
&=1+\frac{1}{2} \mathrm{e}^{\frac{1}{2} k_{1}^{2} \sigma_{\|}^{2}-\mathrm{i} k_{1} q} \operatorname{erfc}\left(\frac{q}{\sqrt{2} \sigma_{\|}}\right)-\frac{1}{2} \operatorname{erfc}\left(\frac{q+\mathrm{i} k_{1} \sigma_{\|}^{2}}{\sqrt{2} \sigma_{\|}}\right),
\end{aligned}
$$

where $\operatorname{erfc}(z)$ is the complementary error function (extended to the complex plane),

$\operatorname{erfc}(z)=\frac{2}{\sqrt{\pi}} \int_{z}^{\infty} \mathrm{d} t \mathrm{e}^{-t^{2}}$

Then, substituting into Eqs. (35) and (7), we can see that the density power spectrum of the "sticky model", $P_{\text {sticky }}(k)$, is equal to the usual Zeldovich power spectrum obtained in Sect. 2.1 plus a correction term $P_{\text {s.c. }}(k)$,

$P_{\text {sticky }}(k)=P_{\text {Zel }}(k)+P_{\text {s.c. }}(k)$,

with

$$
\begin{aligned}
P_{\text {s.c. }}(k)= & \frac{1}{2} \int \frac{\mathrm{d} \boldsymbol{q}}{(2 \pi)^{3}} \mathrm{e}^{-k^{2}\left(1-\mu^{2}\right)\left[\sigma_{v}^{2}-I_{0}(q)-I_{2}(q)\right]} \mathrm{e}^{-q^{2} /\left(2 \sigma_{\|}^{2}\right)} \\
& \times\left\{w\left(\frac{\mathrm{i} q}{\sqrt{2} \sigma_{\|}}\right)-w\left(\frac{\mathrm{i} q-k \mu \sigma_{\|}^{2}}{\sqrt{2} \sigma_{\|}}\right)\right\}
\end{aligned}
$$

Here we introduced Faddeeva's function,

$w(z)=\mathrm{e}^{-z^{2}} \operatorname{erfc}(-\mathrm{i} z)$,

which satisfies the asymptotic expansion (Abramowitz \& Stegun 1970)

$|\arg (z)|<\frac{3 \pi}{4}, z \rightarrow \infty: \quad w(\mathrm{i} z) \sim \frac{1}{\sqrt{\pi} z}\left(1-\frac{1}{2 z^{2}}+..\right)$.

Expression (42) also reads as

$$
\begin{aligned}
P_{\text {s.c. }}(k)= & \int_{0}^{\infty} \frac{\mathrm{d} q q^{2}}{(2 \pi)^{2}} \mathrm{e}^{-q^{2} /\left(2 \sigma_{\|}^{2}\right)} \int_{0}^{1} \mathrm{~d} \mu \mathrm{e}^{-k^{2}\left(1-\mu^{2}\right)\left[\sigma_{v}^{2}-I_{0}-I_{2}\right]} \\
& \times \operatorname{Re}\left\{w\left(\frac{\mathrm{i} q}{\sqrt{2} \sigma_{\|}}\right)-w\left(\frac{\mathrm{i} q-k \mu \sigma_{\|}^{2}}{\sqrt{2} \sigma_{\|}}\right)\right\} .
\end{aligned}
$$

As expected, we can check on Eqs. (42) and (45), using the behavior (44), that the correction due to shell-crossing effects is nonperturbative, in the sense that, because of the term $\mathrm{e}^{-q^{2} /\left(2 \sigma_{\|}^{2}\right)}$, its expansion over powers of the amplitude of the linear power spectrum is identically zero ${ }^{4}$.

\footnotetext{
${ }_{4}$ This is not a large-scale or early-time expansion, which would require knowledge of the shape of $P_{\mathrm{L}}(k)$. As for the standard perturbative expansions of Sects. 2.2 and 2.3, one introduces for instance an auxiliary parameter $\lambda$, such as $\delta_{\mathrm{L}} \rightarrow \lambda \delta_{\mathrm{L}}$ or $P_{\mathrm{L}} \rightarrow \lambda^{2} P_{\mathrm{L}}$, and looks for an expansion over powers of $\lambda$. Since $\sigma_{\|}^{2} \propto q^{2}$ for $q \rightarrow 0$ if $P_{\mathrm{L}}(k)$ decreases faster than $k^{-3}$ at high $k$, as for CDM power spectra, the integration over $q$ does not transform the exponential factor $\mathrm{e}^{-q^{2} /\left(2 \sigma_{\|}^{2}\right)}$ into anomalous power laws, and the expansion of $P_{\text {s.c. }}(k)$ over powers of the amplitude of the linear power spectrum is indeed identically zero, hence nonperturbative.
} 

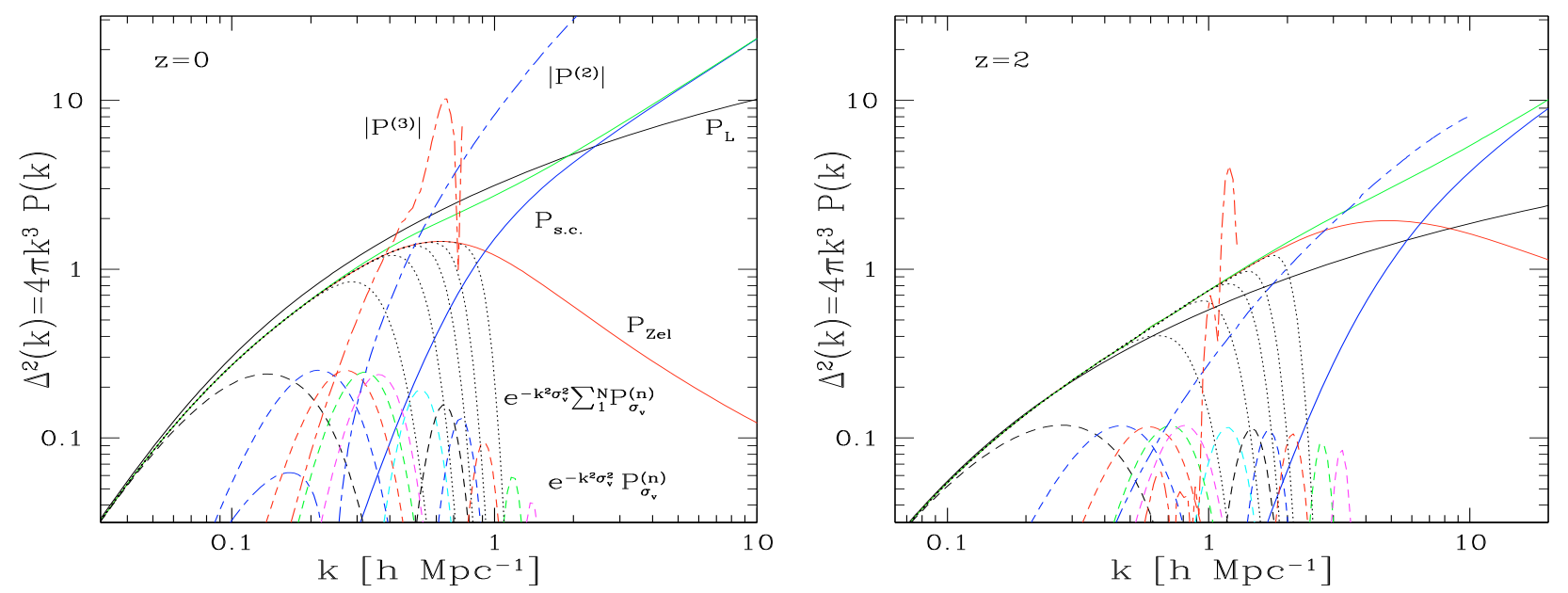

Fig. 1. The power per logarithmic interval of $k$, as defined in Eq. (46), at redshifts $z=0$ (left panel) and $z=2$ (right panel). The solid lines are the linear power spectrum " $P_{\mathrm{L}}$ ", the nonlinear Zeldovich power spectrum " $P_{\text {Zel }}$ ", given by Eq. $(13)$, the nonperturbative correction " $P_{\text {s.c." }}$, given by Eq. (45), and the sum $P_{\text {Zel }}+P_{\text {s.c. }}$ associated with the "sticky model", Eq. (41). The dot-dashed lines, which grow very fast and are only partly drawn, are the absolute values of the standard perturbative terms $\left|P^{(2)}\right|$ and $\left|P^{(3)}\right|$, from Eq. (23). The lower dashed lines are the "renormalized" perturbative terms $\mathrm{e}^{-k^{2} \sigma_{v}^{2}} P_{\sigma_{v}}^{(n)}$ of the expansion (24), from Eq. (26), for $n=1$ to 5 and $n=10,15,20,30,50$, and 70. The peak moves to higher $k$ as the order $n$ increases. The dotted lines that follow the nonlinear Zeldovich power spectrum until a Gaussian decay are the partial sums $\mathrm{e}^{-k^{2} \sigma_{v}^{2}} \sum_{n=1}^{N} P_{\sigma_{v}}^{(n)}(k)$, with $N=5,10,15,20$, and 30 . All terms are multiplied by the factor $4 \pi k^{3}$ of Eq. (46).

At low $k$ the shell-crossing contribution (45) behaves as $P_{\text {s.c. }}(k) \propto k^{2}$. This agrees with the generic behavior ${ }^{5}$ associated with small-scale redistributions of matter (Peebles 1974). Thus, the "sticky model" provides an explicit example of a nonperturbative power spectrum, which satisfies these generic behaviors.

\section{Numerical results in real space}

We now describe the numerical results we obtain for the Zeldovich and "sticky model" power spectra, Eqs. (13) and (41), as well as their common perturbative expansions. We consider a $\Lambda$ CDM cosmology, with $\Omega_{\mathrm{m}}=0.279, \Omega_{\Lambda}=0.721, \sigma_{8}=$ 0.817 , and $h=0.701$.

\subsection{Logarithmic power}

We show in Fig. 1 the power per logarithmic interval of $k$, defined as

$\Delta^{2}(k)=4 \pi k^{3} P(k)$,

for redshifts $z=0$ and $z=2$. We compare the Zeldovich nonlinear power spectrum (13) with its perturbative expansions (19) and (24) and the nonperturbative correction (45). As is well known, higher order terms of the standard perturbative expansion (19) grow increasingly fast at high $k$ with changes in sign and large cancellations between different orders. The "renormalized" perturbative expansion (24) gives positive terms (see Eq. (25)) that peak on a well-defined range (Crocce \& Scoccimarro 2006b) and are much easier to distinguish. Thus, while we only plot the first three orders of the standard expansion, $P^{(1)}=P_{\mathrm{L}}$ and the absolute values $\left|P^{(2)}\right|$ and $\left|P^{(3)}\right|$, we plot the first five orders of the "renormalized" expansion, $P_{\sigma_{v}}^{(1)}$ to $P_{\sigma_{v}}^{(5)}$,

\footnotetext{
5 As described in Peebles (1974), considering momentum conservation, in addition to matter conservation, leads to a $k^{4}$ tail at low $k$. However, since the "sticky model" (34) does not satisfy momentum conservation but only matter conservation, it gives rise to the $k^{2}$ tail.
}

as well as $P_{\sigma_{v}}^{(10)}, P_{\sigma_{v}}^{(15)}, P_{\sigma_{v}}^{(20)}, P_{\sigma_{v}}^{(30)}, P_{\sigma_{v}}^{(50)}$ and $P_{\sigma_{v}}^{(70)}$ (always multiplied by the factor $4 \pi k^{3}$ of Eq. (46)). As we go to higher orders, contributions become narrower and more densely packed, which implies that as we go deeper in the nonlinear regime, we need increasingly more perturbative terms per logarithmic interval of wavenumber.

We also plot a few partial sums of expansion (24), that is, $\mathrm{e}^{-k^{2} \sigma_{v}^{2}} \sum_{n=1}^{N} P_{\sigma_{v}}^{(n)}(k)$, with $N=5,10,15,20$, and 30 . We can check that they agree with the full nonlinear power spectrum (13) until the wavenumber associated with the peak of $\mathrm{e}^{-k^{2} \sigma_{v}^{2}} P_{\sigma_{v}}^{(N)}$, after which they follow the Gaussian decay associated with the prefactor $\mathrm{e}^{-k^{2} \sigma_{v}^{2}}$. These partial sums are also slightly more efficient than those obtained from the standard expansion (19) at the same order (not shown in the figure).

As expected, the nonperturbative correction (45) is very small on quasi-linear scales, so that there is indeed a range where higher order terms of the perturbative expansions (19) and (24) (i.e. beyond the first order associated with the linear regime) are relevant. At higher $k$, the nonperturbative correction becomes dominant and the perturbative expansions become irrelevant, since one can no longer neglect the physics beyond shell crossing.

In the highly nonlinear regime, we recover the well-known decay of the Zeldovich logarithmic nonlinear power spectrum (Schneider \& Bartelmann 1995; Taylor \& Hamilton 1996; Valageas 2007b). This is because particles escape to infinity after shell crossing (i.e. keep moving on their straight trajectories), and these random trajectories (in the sense of random initial conditions) erase small-scale features in the density field. This is expressed by the Gaussian decaying factor $\mathrm{e}^{-k^{2}\left[\sigma_{v}^{2}-I_{0}-\left(1-3 \mu^{2}\right) I_{2}\right]}$ in Eq. (13), where the quantity within brackets is always positive as can be seen from expression (9). Even though this leads to a decay at high $k$, the falloff is not Gaussian because of the integration over the Lagrangian distance $q$ (Taylor \& Hamilton 1996). In particular, for power-law initial power spectra, $P_{\mathrm{L}}(k) \propto$ $k^{n}$ with $-3<n<-1$, one finds that $\Delta^{2}(k) \propto k^{3(n+3) /(n+1)}$ 
P. Valageas: Impact of shell crossing and scope of perturbative approaches, in real and redshift space
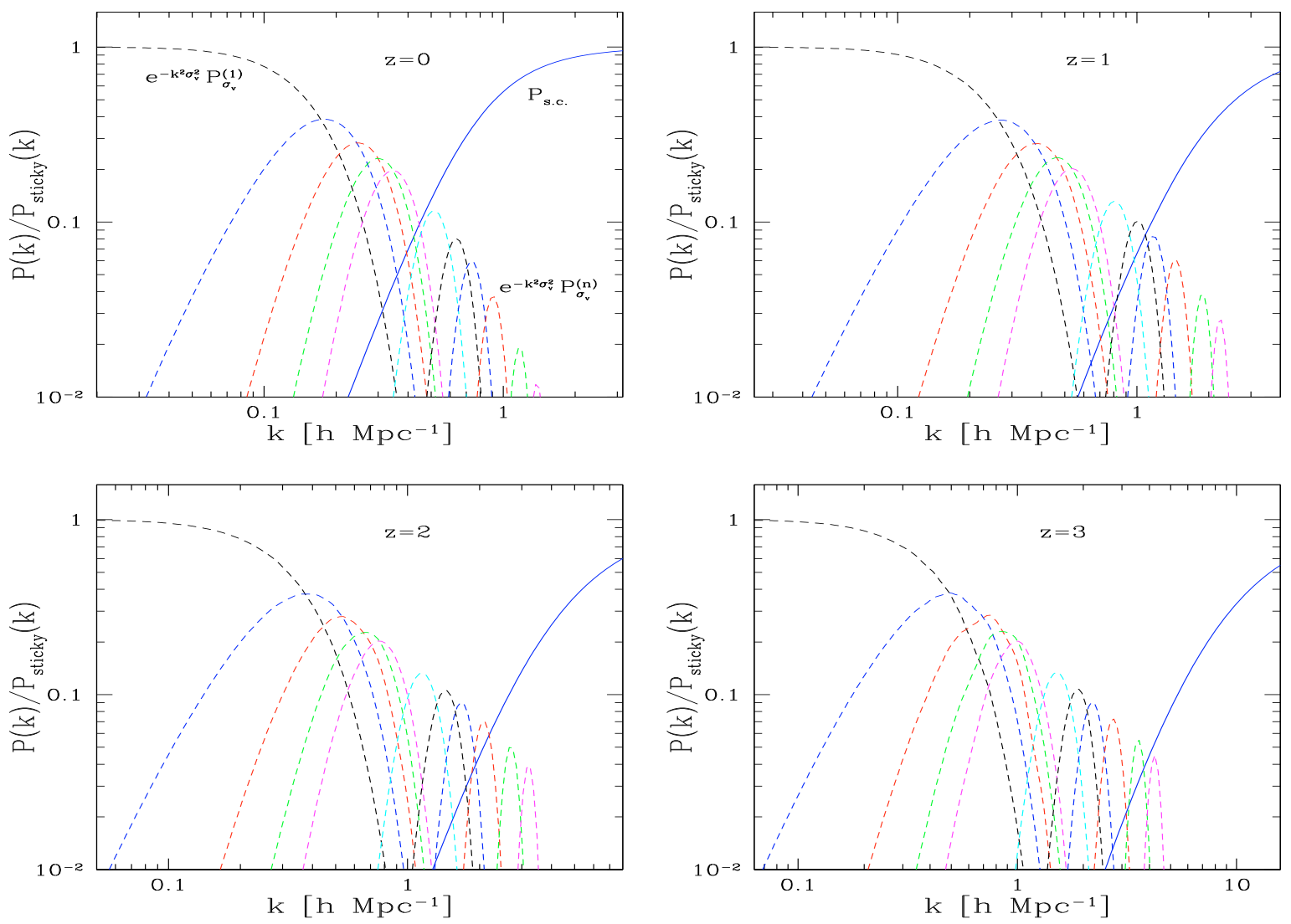

Fig. 2. The dashed lines are the ratios of the "renormalized" perturbative terms of the expansion (24) to the nonlinear "sticky model" power spectrum, $\mathrm{e}^{-k^{2} \sigma_{v}^{2}} P_{\sigma_{v}}^{(n)}(k) / P_{\text {sticky }}(k)$, for $n=1$ to 5 and $n=10,15,20,30,50$, and 70 . The solid line is the ratio of the nonperturbative correction to the nonlinear power spectrum, $P_{\text {s.c. }}(k) / P_{\text {sticky }}(k)$. The four panels correspond to redshifts $z=0,1,2$, and 3 (with a shift of the plot towards higher $k$ at higher $z$ ).

(Valageas 2007b) (the Zeldovich dynamics is not well-defined for $n \geq-1$ because of ultraviolet divergences). Contrary to the gravitational case, the nonlinear power spectrum decreases faster at high $k$ for higher values of $n$. This is due to the greater smearing out of small-scale features by the larger amplitude of the random linear displacements at small wavelengths.

It is interesting to note that, thanks to its shell-crossing correction (45), the nonlinear power spectrum (41) of the "sticky model" does not show this fast decay, and its logarithmic power still increases in the nonlinear regime. This is due to the prescription (34), which in a sense prevents particles from escaping to infinity in one direction, as they stick together. This approximately models the formation of Zeldovich pancakes, associated with collapse along one axis (although there is no precise relationship, since Eq. (34) is only a statistical model and does not consider the "cloud-in-cloud" problem). Thus, small-scale structures are no longer erased, since we keep a trace of planar features. This is expressed by the factor $\left(1-\mu^{2}\right)$ in the exponential argument in expression (45), which suppresses the Gaussian decaying term of the form $\mathrm{e}^{-k^{2}}$ for $\mu \simeq 1$. This gives a width $\Delta \mu \sim k^{-2}$, hence $P_{\text {s.c. }}(k) \sim k^{-2}$ at high $k$, as would be the case for a density field where planar objects are the relevant nonlinear structures (i.e. bidimensional structures, as opposed to pointlike masses or lines for instance). Contrary to the Zeldovich power spectrum there is no dependence on $n$ for the high- $k$ slope, for power-law linear power spectra $P_{\mathrm{L}}(k) \propto k^{n}$ with $-3<n<-1$. This gives the universal asymptote $\Delta_{\text {sticky }}^{2}(k) \propto k$ for the nonlinear logarithmic power of the "sticky model" at high $k$.

\subsection{Perturbative and nonperturbative contributions}

To clearly see the range of scales where perturbative schemes are relevant, we plot in Fig. 2 the ratios of the successive terms of the "renormalized" perturbative expansion (24) with respect to the nonlinear power spectrum of the "sticky model", $\mathrm{e}^{-k^{2} \sigma_{v}^{2}} P_{\sigma_{v}}^{(n)}(k) / P_{\text {sticky }}(k)$, for $n=1$ to 5 , as well as $n=10,15$, $20,30,50$, and 70 . We also plot the ratio $P_{\text {s.c. }}(k) / P_{\text {sticky }}(k)$, to compare with the amplitude of the nonperturbative correction associated with shell-crossing effects. We consider the four redshifts $z=0,1,2$, and 3 . Here we focus on the "renormalized" perturbative expansion (24), rather than on the standard expansion (19), to avoid the interferences brought by the changes in sign and the cancellations between various terms.

We can see that the potential of perturbative expansions grows at higher redshift for a $\Lambda C D M$ power spectrum, because of the change in slope of $P_{\mathrm{L}}(k)$. Thus, at $z=3$ we can go up to the order $n=66$ before the perturbative term becomes subdominant with respect to the nonperturbative correction, while at $z=0$ the crossover takes place at $n=9$, as the term $\mathrm{e}^{-k^{2} \sigma_{v}^{2}} P_{\sigma_{v}}^{(10)}(k)$ is the lowest order one that is fully below the nonperturbative correction $P_{\text {s.c. }}(k)$. This agrees with the observation that in the gravitational case perturbative schemes (and resummation approaches) seem to fare better at higher $z$ (Carlson et al. 2009). We can see that perturbative schemes are relevant over roughly one decade over $k$. Thus, if we require an accuracy of $1 \%$ at $z=0$, linear theory is sufficient up to $k_{1 \text { loop }} \sim 0.033 h \mathrm{Mpc}^{-1}$, while higher order perturbative terms allow reaching $k_{\text {s.c. }} \sim 0.23 h \mathrm{Mpc}^{-1}$. 
Table 1. The dependence on redshift $z$ of the wavenumbers where the ratios $\mathrm{e}^{-k^{2} \sigma_{v}^{2}} P_{\sigma_{v}}^{(2)}(k) / P_{\text {sticky }}(k)$ (at $\left.k_{\text {lloop }}\right)$ and $P_{\text {s.c. }}(k) / P_{\text {sticky }}(k)\left(\right.$ at $\left.k_{\text {s.c. }}\right)$ reach $1 \%, 10 \%$, and $50 \%$.

\begin{tabular}{ccccc}
\hline \hline$z$ & $\%$ & $k_{1 \text { loop }}\left[h \mathrm{Mpc}^{-1}\right]$ & $k_{\text {s.c. }}\left[h \mathrm{Mpc}^{-1}\right]$ & $n_{\text {s.c. }}$ \\
\hline & $1 \%$ & 0.033 & 0.23 & \\
0 & $10 \%$ & 0.082 & 0.45 & 9 \\
& $50 \%$ & & 0.9 & \\
\hline \multirow{3}{*}{1} & $1 \%$ & 0.043 & 0.44 & \\
& $10 \%$ & 0.11 & 1.1 & 18 \\
& $50 \%$ & & 2.2 & \\
\hline \multirow{2}{*}{2} & $1 \%$ & 0.057 & 1.2 & \\
& $10 \%$ & 0.14 & 2.3 & 37 \\
& $50 \%$ & & 6.4 & \\
\hline \multirow{3}{*}{3} & $1 \%$ & 0.07 & 2.2 & \\
& $10 \%$ & 0.18 & 5.2 & 66 \\
& $50 \%$ & & 10.4 & \\
\hline
\end{tabular}

Notes. The last column gives the last order $n_{\text {s.c. }}$ of the "renormalized" perturbative expansion that is not fully below the nonperturbative term.

At higher $k$ one must take the nonperturbative correction associated with shell-crossing effects into account, which implies going beyond the fluid approximation and requires new approaches.

To help the reader, we give in Table 1 the wavenumber $k_{1 \text { loop }}$, below which the linear term is enough to reach a $1 \%$ or $10 \%$ accuracy, for the four redshifts shown in Fig. 2. We also give the wavenumber $k_{\text {s.c. }}$ beyond which the nonperturbative correction is required to reach an accuracy of $1 \%, 10 \%$, or $50 \%$ (in units of $P_{\text {sticky }}(k)$ ). Thus, the interval $\left[k_{1 \text { loop }}, k_{\text {s.c. }}\right]$ gives the range where perturbation theories based on the fluid description are relevant. Of course, this range shifts to higher $k$ at higher redshift. It is interesting to note that this range is also broader at higher redshift, as the slope of the CDM linear power spectrum on the relevant scales changes slowly. The last column gives the last order, $n_{\text {s.c. }}$, of the "renormalized" perturbative expansion that is not fully below the nonperturbative term. As noticed above in Fig. 2, this expansion order is significantly higher at higher redshift. This corresponds to a greater potential for perturbative schemes. However, $n_{\text {s.c. }}$ grows faster than the logarithmic width of the perturbative range, [ $\ln k_{1 \text { loop }}, \ln k_{\text {s.c. }}$. Indeed, as shown in Fig. 2, peaks associated with higher order perturbative terms are increasingly narrow on the $\ln k$ axis. This implies that to multiply the upper wavenumber $k$, defined by a fixed accuracy, by a given amount, one needs to add an increasingly greater number of new perturbative terms.

In practice, we do not expect that perturbative terms will be computed up to such high orders, since in the case of the gravitational dynamics this would involve multidimensional integrals that are beyond the reach of current numerical possibilities. However, resummation schemes allow one to consider parts of such higher order terms (actually, an infinite number of diagrams that contribute to terms of all orders). Then, the hope is that such methods can efficiently sum most of the contributions of higher order terms and accelerate the convergence on weakly nonlinear scales. The comparison displayed in Fig. 2 shows the potential of such methods (i.e. the best result one can obtain, for the "sticky model" considered here), which appears to be quite significant at higher redshifts, $z \geq 1$.

Since the Zeldovich dynamics provides a reasonable approximation of the gravitational dynamics down to weakly nonlinear scales (Coles et al. 1993; Pauls \& Melott 1995), and its accuracy can be improved by implementing the "adhesion model" that only differs after shell crossing (Weinberg \& Gunn 1990; Melott et al. 1994; Sathyaprakash et al. 1995), we can expect that to a large extent these results still apply to the gravitational case. In particular, while we find that at $z=0$ the nonperturbative correction to the density power spectrum is around $1 \%$ at $k \sim 0.23 h \mathrm{Mpc}^{-1}$ and $50 \%$ at $k \sim 0.9 h \mathrm{Mpc}^{-1}$, Afshordi (2007) finds the wavenumbers $k \sim 0.1 h \mathrm{Mpc}^{-1}$ and $k \sim 0.85 h \mathrm{Mpc}^{-1}$ with a phenomenological "sticky halo model". It is comforting that these two very different approaches give similar results. Then, the property that the range where perturbative schemes are relevant is greater at $z=3$ than at $z=0$, with a higher order $n_{\text {s.c., }}$ should remain valid.

This is confirmed by the analysis of unified models that combine perturbation theories with halo models, for the gravitational case. Thus, as shown in Valageas \& Nishimichi (2010), at higher redshift the intermediate range, where the power spectrum departs from one-loop perturbation theory but is not yet well described by the "one-halo" contribution, becomes wider. There, the "one-halo" contribution plays the role of the nonperturbative contribution (45), as it is also fully nonperturbative and decays at low $k$ as $k^{2}$ (if we only consider matter conservation, see the discussion in Valageas \& Nishimichi 2010). Then, the broadening of this intermediate range also suggests that higher orders of perturbation theory become relevant, as explicitly found in Figs. 1 and 2.

In the context of cosmological reconstruction ${ }^{6}$, a comparison with $N$-body simulations (Mohayaee et al. 2006) shows that the Monge-Ampère-Kantorovich method is able to recover the nonlinear displacement field down to $\sim 3 h^{-1} \mathrm{Mpc}$ at $z=0$, which corresponds roughly to $k \sim 2 h \mathrm{Mpc}^{-1}$. We can see from Table 1 that this is a very good result, as going to smaller scales requires taking shell crossing into account (in fact, at $k=2 \mathrm{~h} \mathrm{Mpc}^{-1}$ nonperturbative corrections have already started to dominate). Thus, the Monge-Ampère-Kantorovich method appears to be close to optimal at $z=0$, because it goes as far as any scheme that disregards shell-crossing effects can be expected to go. This can be understood partly from the fact that relatively few orders of perturbations theory are relevant at $z=0$ (since $n_{\text {s.c. }}=9$ ), so that it may not help much to explicitly include the effects of higher order terms.

Reconstruction techniques are also used to sharpen the acoustic peak of the real-space correlation function or to restore the harmonics of the oscillations of the power spectrum, in order to improve cosmological distance measurements and constraints on dark energy (Eisenstein et al. 2007; Seo et al. 2010). Then, one can read in Table 1 the wavenumber $k_{\text {s.c. }}$ up to which one can hope to recover these baryon acoustic oscillations. At $z=0$

\footnotetext{
6 There, the problem is to reconstruct the past dynamical history of a given region of the sky from the knowledge of its present density field. A key observation is that in the linear growing mode, the velocity field is curlfree and related to the linear gravitational potential by a relation of the form $\boldsymbol{v}_{\mathrm{L}} \propto-\nabla \Phi_{\mathrm{L}}$, while the linear density field is given by the Poisson equation, $\delta_{\mathrm{L}} \propto \Delta \Phi_{\mathrm{L}}$. Then, the number of unknowns (the linear gravitational potential) is equal to the number of constraints (the present nonlinear density field), so that it makes sense to look for a reconstruction defined by the nonlinear density field alone (but this does not ensure uniqueness). The Monge-Ampère-Kantorovich method makes use of an additional assumption, that the Lagrangian map, $\boldsymbol{q} \mapsto \boldsymbol{x}$, can be derived from a convex potential $\varphi(\boldsymbol{q})$, as in Eq. (29), to derive a unique solution to the "displacement reconstruction", i.e. the inverse map $\boldsymbol{x} \mapsto \boldsymbol{q}$ (Brenier et al. 2003; Mohayaee et al. 2006). Such methods cannot describe multi-streaming, and by estimating the scale where shell crossing effects can no longer be neglected, we can evaluate the scale down to which these reconstruction schemes can apply.
} 
we can see that present schemes, which are based on the linear displacement field and manage to reach $\sim 0.2 \mathrm{~h} \mathrm{Mpc}^{-1}$ (not necessarily for the amplitude but at least for the shape and position of the oscillations) are not far from the upper bound, as could be expected from only a few orders of perturbation theory being relevant $\left(n_{\text {s.c. }}=9\right)$. At $z=3$ it seems that one could go much beyond present schemes (which do not go much farther than $0.25 \mathrm{~h} \mathrm{Mpc}^{-1}$ ), in agreement with $n_{\text {s.c. }}=66$, which means that higher orders of perturbation theory are relevant. However, for the specific purpose of measuring cosmological distances from the baryon acoustic oscillations, the potential is limited by the relative amplitude of the oscillations of the linear power spectrum itself decreasing at higher $k$, so that even a very good reconstruction would not greatly enhance the signal-to-noise ratio. Nevertheless, pushing to higher orders (e.g., through resummation schemes) remains useful for other purposes, such as weaklensing studies.

\subsection{Rise of power at the transition}

The increase of power on the transition scale to nonlinearity shown in Fig. 1 (especially in the left panel at $z=0$ ) is reminiscent of a similar feature observed for the gravitational dynamics (Hamilton et al. 1991; Peacock \& Dodds 1996). This is usually interpreted from a Lagrangian point of view inspired by the spherical collapse dynamics. Thus, Padmanabhan (1996) argues that, on these intermediate scales, one has $\bar{\xi}(x) \propto \bar{\xi}_{\mathrm{L}}(q)^{3}$ in real space with $q^{3}=x^{3}(1+\bar{\xi})$. More generally, in Fourier space one writes for the nonlinear power per logarithmic interval of wavenumber, $\Delta^{2}(k)$, the parametric system (Peacock \& Dodds 1996)

$k_{\mathrm{L}}=\left[1+\Delta^{2}(k)\right]^{-1 / 3} k$,

$\Delta^{2}(k)=f\left[\Delta_{\mathrm{L}}^{2}\left(k_{\mathrm{L}}\right)\right]$,

with a function $f$ to be determined. These relations express the conservation of matter, since the Lagrangian scale $q \sim 1 / k_{\mathrm{L}}$ collapses down to the Eulerian scale $x \sim 1 / k$. The linear regime implies that $f(x) \simeq x$ for $x \ll 1$, whereas in the highly nonlinear regime the stable-clustering ansatz (Peebles 1982) gives the scaling $f(x) \sim x^{3 / 2}$ for $x \gg 1$. At the transition one observes a sharper growth, which is consistent with $f(x) \sim x^{3}$ (Padmanabhan 1996). In practice, one builds a fitting formula for $f(x)$ to match numerical simulations and to account for the dependence on the shape of the linear power spectrum.

In any case, such models usually estimate the shape of the nonlinear two-point correlation function or of the nonlinear power spectrum by considering the collapse of a "typical" overdensity (Padmanabhan 1996; Valageas \& Schaeffer 1997) (or merely obtaining $f(x)$ from simulations without further interpretation). It is interesting to note that this collapse also takes place within the Zeldovich dynamics studied here. In particular, the function $\delta=\mathcal{F}\left(\delta_{\mathrm{L}}\right)$ that describes the spherical collapse is no longer given by cycloids (Peebles 1980) but by the simple expression $\mathcal{F}\left(\delta_{\mathrm{L}}\right)=1 /\left(1-\delta_{\mathrm{L}} / 3\right)^{3}-1($ Bernardeau \& Kofman 1995; Valageas 2009). (Collapse to a point is delayed from $\delta_{\mathrm{c}} \simeq 1.686$ to $\delta_{\mathrm{c}}=3$, since the motion does not accelerate as the gravitational potential well becomes deeper.)

We show in Fig. 3 the functions $\Delta^{2}(k)=f\left[\Delta_{\mathrm{L}}^{2}\left(k_{\mathrm{L}}\right)\right]$ defined by the system (47), (48) for the Zeldovich dynamics and the "sticky model". Indeed, from the knowledge of the nonlinear power $\Delta^{2}(k)$ we obtain the Lagrangian wavenumber $k_{\mathrm{L}}$

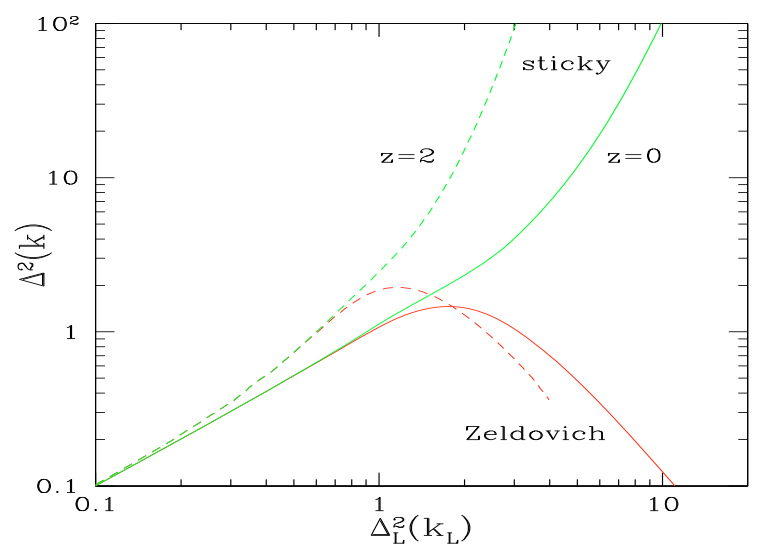

Fig. 3. The nonlinear power per logarithmic interval of wavenumber, $\Delta^{2}(k)$, as a function of the linear power $\Delta_{\mathrm{L}}^{2}\left(k_{\mathrm{L}}\right)$, at the Lagrangian wavenumber $k_{\mathrm{L}}$ defined by Eq. (47). The lower curves are obtained from the Zeldovich power spectrum (13), while the upper curves correspond to the power spectrum (41) of our "sticky model". The solid lines are for the redshift $z=0$ and the dashed lines for $z=2$.

from Eq. (47) and next $\Delta_{\mathrm{L}}^{2}\left(k_{\mathrm{L}}\right)$. For a given Eulerian wavenumber $k$, the Lagrangian wavenumbers $k_{\mathrm{L}}$ obtained for both models, Zeldovich dynamics and "sticky model", are different. Then, Fig. 3 shows that, within the Zeldovich dynamics, this growth of the nonlinear density contrast through the spherical collapse is not sufficient to build up the increase in power on these mildly nonlinear scales, although one can see a modest rise at $z=2$. Indeed, the shape of the nonlinear power spectrum is quickly governed by the decay that takes place at higher $k$, due to the escape of particles beyond shell crossing that erases small-scale features, as recalled in Sect. 3.1. In contrast, such a growth is clearly seen in the "sticky model".

This means that such arguments, based on the evolution of a "typical" overdensity through the spherical collapse dynamics, are not good enough to explain the shape of the power spectrum on these scales since they clearly fail for the Zeldovich dynamics. Thus, one needs to consider the behavior of a whole range of typical density fluctuations (i.e., truly perform the Gaussian average over the initial conditions), which includes a significant number of configurations where shell crossing has already taken place. Then, one cannot neglect the dependence on their behavior after shell crossing, and whereas the free escape associated with the Zeldovich dynamics is sufficient to erase any growth of power on the transition scale, a trapping of particles allows such a growth. In the "sticky model" studied here, this trapping after shell crossing is a simple sticking along one direction, as defined in (34), which eventually leads to a universal $k^{-2}$ tail at very high $k$ for $P_{\text {sticky }}(k)$, as noticed in Sect. 3.1. In terms of the function $f\left[\Delta_{\mathrm{L}}^{2}\left(k_{\mathrm{L}}\right)\right]$, this corresponds to a large- $x$ scaling $f_{\text {sticky }}(x) \propto x^{3 /[2(n+3)]}$ from the system (47), (48).

In the gravitational case, this regime corresponds to the virialization within $3 \mathrm{D}$ bound structures, with an asymptotic tail at high $k$ that is poorly known. In particular, although overdensities seem to form halos with an almost universal profile (Navarro et al. 1997), there is no first-principle derivation of the high- $k$ exponent of the density power spectrum, and its degree of universality is not well known. However, especially in the case of CDM-like power spectra that decay as $P_{\mathrm{L}}(k) \propto k^{-3}$ at high $k$, halos are rather smooth with a low amount of substructures. Then, the nonlinear logarithmic power spectrum does not seem to show 
a strong high- $k$ tail, such as the universal forms $\Delta^{2}(k) \propto k^{3}$, $\propto k^{2}$ or $\propto k^{1}$, associated with pointlike masses, lines, or sheets, but appears to be consistent with a logarithmic or shallow slope asymptote (Smith et al. 2003). This means that the steep rise in the function $\Delta^{2}(k)=f\left[\Delta_{\mathrm{L}}^{2}\left(k_{\mathrm{L}}\right)\right]$ observed in the intermediate regime, $1<\Delta^{2}(k)<100$, breaks down at $\Delta^{2}(k) \sim 100$ to reach this slow growth at higher $k$, in contrast to the "sticky model" considered here where there is a regular transition from the linear regime, where $f_{\text {sticky }}(x) \sim x$, to the highly nonlinear regime, where $f_{\text {sticky }}(x) \propto x^{3 /[2(n+3)]}$.

On the other hand, in agreement with the fact that the linear density contrast associated with full spherical collapse to a point is delayed from $\delta_{\mathrm{c}} \simeq 1.686$ to 3 , the rise obtained in Fig. 3 at $\Delta_{\mathrm{L}}^{2}(k) \sim 1$ appears at somewhat higher values of $\Delta_{\mathrm{L}}^{2}(k)$ than would be the case for the gravitational dynamics, as can be checked by comparison with the results of $N$-body simulations (Smith et al. 2003). In this respect, the spherical collapse captures some of the properties of the early rise of $\Delta^{2}(k)$.

\section{Redshift-space power spectrum}

The density field observed from galaxy surveys is distorted by their peculiar velocities, which introduce an error in the measure of their radial position (Jackson 1972; Kaiser 1987). Indeed, both the mean Hubble flow and the peculiar radial velocity of a galaxy contribute to the redshift of the observed emission and absorption lines. On the other hand, the measure of the angular positions on the sky are not influenced by these peculiar velocities. In a "plane-parallel" approximation, where the line of sight has a fixed direction $\boldsymbol{e}_{z}$, radial and angular modes correspond to longitudinal and transverse wavenumbers $k_{\|}$and $\boldsymbol{k}_{\perp}$. Then, the power spectrum measured along the transverse direction (i.e. $k_{\|}=0$ ) is not affected by these "redshift distortions" and is equal to the real-space power spectrum considered in the previous sections, whereas the power spectrum measured along the longitudinal direction (i.e. $\boldsymbol{k}_{\perp}=0$ ) is modified. In particular, this means that the power spectrum is no longer isotropic.

This implies that some additional information is contained in the redshift-space power spectrum, since by comparing the longitudinal and transverse components, we can derive some information on the velocity field and obtain further constraints on cosmology. For instance, within linear theory, comparing the redshift-space power spectrum over several directions (Kaiser 1987) or expanding its angular dependence over Legendre polynomials and comparing the first few multipoles (Hamilton 1992), one can constrain the ratio $f$ of the linear growth rate to its value in a critical density universe, hence $\Omega_{\mathrm{m}}$ since $f \simeq \Omega_{\mathrm{m}}^{0.6}$. On the other hand, while clustering along the transverse directions of statistical standard rulers such as BAO gives the angular distance, real-space clustering along the longitudinal direction gives the Hubble rate, so that complementary information can be derived from both directions, and one can use the longitudinal/transverse ratio to perform the Alcock-Paczynski test (Alcock \& Paczynski 1979).

Thus, it is interesting to extend the analysis presented in the previous sections to the redshift-space power spectrum. Rather than expanding on multipoles, we focus on the clustering along the radial direction, as compared with the transverse directions. Of course, another effect that comes into play in galaxy surveys is the bias, which may show some scale dependence. However, we do not study this effect here, as this is a rather different process.

\subsection{Nonlinear Zeldovich power spectrum in redshift space}

We first recall the nonlinear redshift-space power spectrum associated with the Zeldovich dynamics. The redshift-space coordinate $s$ of a galaxy is

$\boldsymbol{s}=\boldsymbol{x}+\frac{\boldsymbol{e}_{z} \cdot \boldsymbol{v}}{a H} \boldsymbol{e}_{z}$

where $\boldsymbol{x}$ is its comoving position, $\boldsymbol{v}=a \dot{\boldsymbol{x}}$ its peculiar velocity, and $\boldsymbol{e}_{z}$ the unit vector of the line of sight. Within the Zeldovich dynamics (1) the peculiar velocity is

$\boldsymbol{v}=a \dot{\Psi}_{\mathrm{L}}=\frac{a \dot{D}}{D} \boldsymbol{\Psi}_{\mathrm{L}}$,

leading to

$\boldsymbol{s}(\boldsymbol{q}, t)=\boldsymbol{q}+\boldsymbol{\Psi}_{\mathrm{L}}+f\left(\boldsymbol{e}_{z} \cdot \boldsymbol{\Psi}_{\mathrm{L}}\right) \boldsymbol{e}_{z}$,

where $D$ is the linear growth factor and $f(z)=\mathrm{d} \ln D / \mathrm{d} \ln a$. In the following we use a "plane-parallel" approximation and we denote the longitudinal and transverse directions to the line of sight by the subscripts $\|$ and $\perp$. Thus we have

$s_{\|}=q_{\|}+(1+f) \Psi_{L \|}, \quad s_{\perp}=q_{\perp}+\Psi_{L \perp}$.

The conservation of matter reads again as $\rho^{s}(\boldsymbol{s}) \mathrm{d} \boldsymbol{s}=\bar{\rho} \mathrm{d} \boldsymbol{q}$, where we denote the redshift-space quantities by a superscript " $s$ ", and as in Eqs. (3)-(7) the redshift-space power spectrum reads as

$P^{s}(\boldsymbol{k})=\int \frac{\mathrm{d} \boldsymbol{q}}{(2 \pi)^{3}}\left\langle\mathrm{e}^{\mathrm{i} \boldsymbol{k} \cdot[s(\boldsymbol{q})-s(0)]}\right\rangle$.

Following Taylor \& Hamilton (1996), it is convenient to introduce the vector $\boldsymbol{K}$, which is the wavevector $\boldsymbol{k}$ stretched by $(1+f)$ along the line of sight,

$\boldsymbol{K}=(1+f) k_{\|} \boldsymbol{e}_{z}+\boldsymbol{k}_{\perp}$.

Then Eq. (53) writes as (see Eq. (13))

$\begin{aligned} P^{s}(\boldsymbol{k}) & =\int \frac{\mathrm{d} \boldsymbol{q}}{(2 \pi)^{3}} \mathrm{e}^{\mathrm{i} \boldsymbol{k} \cdot \boldsymbol{q}}\left\langle\mathrm{e}^{\mathrm{i} \boldsymbol{K} \cdot\left[\boldsymbol{\Psi}_{\mathrm{L}}(\boldsymbol{q})-\boldsymbol{\Psi}_{\mathrm{L}}(0)\right]}\right\rangle \\ & =\int \frac{\mathrm{d} \boldsymbol{q}}{(2 \pi)^{3}} \mathrm{e}^{\mathrm{i} \boldsymbol{k} \cdot \boldsymbol{q}} \mathrm{e}^{-K^{2}\left[\sigma_{v}^{2}-I_{0}(q)-\left(1-3 \mu_{K q}^{2}\right) I_{2}(q)\right]},\end{aligned}$

where $\mu_{K q}=(\boldsymbol{K} \cdot \boldsymbol{q}) /(K q)$. Using spherical coordinates about the vector $\boldsymbol{K}$, expanding parts of the exponentials and using Eq. (14) we obtain

$$
\begin{aligned}
P^{s}(\boldsymbol{k})= & \int_{0}^{\infty} \frac{\mathrm{d} q q^{2}}{2 \pi^{2}} \mathrm{e}^{-K^{2}\left[\sigma_{v}^{2}-I_{0}(q)+2 I_{2}(q)\right]} \sum_{\ell, m=0}^{\infty} \frac{(\ell+m) !}{\ell ![(2 m) ! !]^{2}} \\
& \times\left(\frac{6 K^{2} I_{2}(q)}{k q \mu_{K k}}\right)^{\ell}\left(\frac{2 k q\left(\mu_{K k}^{2}-1\right)}{\mu_{K k}}\right)^{m} j_{\ell+m}\left(k q \mu_{K k}\right),
\end{aligned}
$$

with

$\mu_{k}=\frac{\boldsymbol{k} \cdot \boldsymbol{e}_{z}}{k}, \quad \mu_{K k}=\frac{\boldsymbol{K} \cdot \boldsymbol{k}}{K k}=\frac{1+f \mu_{k}^{2}}{\sqrt{1+\mu_{k}^{2}\left(2 f+f^{2}\right)}}$

$K^{2}=k^{2}\left[1+\mu_{k}^{2}\left(2 f+f^{2}\right)\right]$.

Expression (56) holds for any wavenumber $\boldsymbol{k}$ and shows how the redshift-space power spectrum depends on both $k$ and $\mu_{k}$. 
As recalled above and as is obvious from Eq. (52), the redshiftspace power spectrum for wavevectors perpendicular to the line of sight is equal to the real-space power spectrum,

$P_{\perp}^{s}\left(k_{\perp}\right) \equiv P^{s}\left(\boldsymbol{k}_{\perp}\right)=P\left(k_{\perp}\right)$,

and we can check that we recover Eqs. (15) from (56) for $\mu_{k}=0$. For longitudinal wavevectors, we simply have $\boldsymbol{K}=(1+f) \boldsymbol{k}$, so that Eq. (55) simplifies as

$$
\text { for } \begin{aligned}
\boldsymbol{k} & =k_{\|} \boldsymbol{e}_{z}: \quad P_{\|}^{S}\left(k_{\|}\right) \equiv P^{S}\left(k_{\|} \boldsymbol{e}_{z}\right) \\
& =\int \frac{\mathrm{d} \boldsymbol{q}}{(2 \pi)^{3}} \mathrm{e}^{\mathrm{i} \boldsymbol{k} \cdot \boldsymbol{q}} \mathrm{e}^{-k^{2}(1+f)^{2}\left[\sigma_{v}^{2}-I_{0}(q)-\left(1-3 \mu^{2}\right) I_{2}(q)\right]},
\end{aligned}
$$

where $\mu=(\boldsymbol{k} \cdot \boldsymbol{q}) /(k q)=q_{\|} / q$, as in Eq. (13). Thus we see at once that, along the line of sight, the effect of redshift distortions is merely to multiply the amplitude of the linear power spectrum by a factor $(1+f)^{2}$; that is, we can absorb the factor $(1+f)^{2}$ into $\sigma_{v}^{2}, I_{0}$ and $I_{2}$, or more simply into $P_{\mathrm{L}}(k)$, as was obvious from Eq. (52). Therefore, we directly obtain from Eq. (15) the expression

$$
\begin{aligned}
P_{\|}^{S}(k)= & \int_{0}^{\infty} \frac{\mathrm{d} q q^{2}}{2 \pi^{2}} \mathrm{e}^{-k^{2}(1+f)^{2}\left[\sigma_{v}^{2}-I_{0}(q)+2 I_{2}(q)\right]} \\
& \times \sum_{\ell=0}^{\infty}\left(\frac{6 k(1+f)^{2} I_{2}(q)}{q}\right)^{\ell} j_{\ell}(k q),
\end{aligned}
$$

since we only need to multiply each term that involves $P_{\mathrm{L}}(k)$ by a factor $(1+f)^{2}$. In a similar fashion, the standard perturbative expansion (19) becomes

$P_{\|}^{s}(k)=\sum_{n=1}^{\infty} P_{\|}^{s(n)}(k)$ with $P_{\|}^{s(n)}(k)=(1+f)^{2 n} P^{(n)}(k)$,

whereas the "renormalized" perturbative expansion (24) becomes

$P_{\|}^{s}(k)=\mathrm{e}^{-k^{2}(1+f)^{2} \sigma_{v}^{2}} \sum_{n=1}^{\infty} P_{\| \sigma_{v}}^{s(n)}(k)$

with $P_{\| \sigma_{v}}^{s(n)}(k)=(1+f)^{2 n} P_{\sigma_{v}}^{(n)}(k)$.

Thus, each order $n$ of the perturbative expansions gets multiplied by a factor $(1+f)^{2 n}$ as we go from real space to redshift space, for modes that are aligned with the line of sight. In particular, at lowest order we have $P_{\|}^{s}(k)=(1+f)^{2} P_{\mathrm{L}}(k)+\ldots$, so that we recover the well-known boost factor of Kaiser (1987), associated with the infall of galaxies within gravitational potential wells. On the other hand, the factor $(1+f)^{2}$ also sharpens the Gaussian damping prefactor in the expansion (63).

\section{2. "Sticky model" nonperturbative correction}

We now turn to the nonperturbative correction associated with the "sticky model" introduced in Sect. 2.4. Again we focus on the redshift-space power spectrum $P_{\|}^{s}(k)$ for wavenumbers that are parallel to the line of sight. From Eq. (53) we now need to compute the average $\left\langle\mathrm{e}^{\mathrm{i} k \cdot \Delta s}\right\rangle_{q}$. Before shell crossing, or more precisely, as long as $\Delta x_{L 1}>0$ for a Lagrangian-space separation vector along the $\boldsymbol{e}_{1}$-axis, the "sticky model" follows the Zeldovich dynamics,

$$
\begin{aligned}
& \boldsymbol{k}= k \boldsymbol{e}_{z}, \boldsymbol{q}=q \boldsymbol{e}_{1}: \\
& \quad \text { if } \Delta \Psi_{L 1}>-q: \boldsymbol{k} \cdot \Delta \boldsymbol{s}=\boldsymbol{k} \cdot \boldsymbol{q}+(1+f) \boldsymbol{k} \cdot \boldsymbol{\Psi}_{\mathrm{L}},
\end{aligned}
$$

whereas once $\Delta x_{L 1}<0$ we affect the same position, $\Delta x_{1}=0$, and the same peculiar velocity, $\Delta v_{1}=0$, along the $\boldsymbol{e}_{1}$-axis, for both particles as in (34), whence

$$
\text { if } \begin{aligned}
\Delta \Psi_{L 1}<-q: \Delta s_{1}=0, \text { hence } \\
\boldsymbol{k} \cdot \Delta \boldsymbol{s}=(1+f)\left[k_{2} \Psi_{L 2}+k_{3} \Psi_{L 3}\right] .
\end{aligned}
$$

(The line-of-sight axis $\boldsymbol{e}_{z}$ and the axis $\boldsymbol{e}_{1}$ of the Lagrangian separation vector $\boldsymbol{q}$ are not related.) At the shell-crossing time we have a discontinuity in the redshift-space separation $\Delta \boldsymbol{s}$, since $\Delta s_{1}$ jumps from $-f q$ to zero. Indeed, just before contact, the two particles move closer, with the finite relative velocity $\Delta v_{1}=-(a \dot{D} / D) q$, while after collision their relative velocity is set to zero. This should actually be understood in a loose sense, since within the "sticky model" we consider neither transverse directions nor the cloud-in-cloud problem. Then, as in Sect. 2.4 the redshift-space power spectrum of the "sticky model" reads as

$P_{\text {sticky }}^{s}(\boldsymbol{k})=P_{\text {Zel }}^{s}(\boldsymbol{k})+P_{\text {s.c. }}^{s}(\boldsymbol{k})$,

and from Eqs. (65), (66) we obtain for wavenumbers along the line of sight

$$
\begin{aligned}
P_{\| \text {s.c. }}^{s}(k)= & \int \frac{\mathrm{d} q q^{2}}{(2 \pi)^{2}} \mathrm{e}^{-q^{2} /\left(2 \sigma_{\|}^{2}\right)} \int_{0}^{1} \mathrm{~d} \mu \mathrm{e}^{-k^{2}(1+f)^{2}\left(1-\mu^{2}\right)\left[\sigma_{v}^{2}-I_{0}-I_{2}\right]} \\
& \times \operatorname{Re}\left\{\omega\left(\frac{\mathrm{i} q}{\sqrt{2} \sigma_{\|}}\right)-\mathrm{e}^{-\mathrm{i} k f q \mu} w\left(\frac{\mathrm{i} q-k(1+f) \mu \sigma_{\|}^{2}}{\sqrt{2} \sigma_{\|}}\right)\right\} .
\end{aligned}
$$

As compared with Eq. (45), the new exponential factor $\mathrm{e}^{-\mathrm{i} k f q \mu}$ is due to the discontinuity of $\Delta s$ at $\Delta \Psi_{L 1}=-q$, associated with shell crossing within the "sticky model". The factors $(1+f)$ that multiply the longitudinal wavenumber $k$ could be expected from Eqs. (65), (66).

\subsection{Redshift-space logarithmic power}

As for the real-space Fig. 1, we show in Fig. 4 our numerical results for the redshift-space logarithmic power, for longitudinal wavenumbers $\boldsymbol{k}$, defined as

$\Delta_{\|}^{2 s}(k)=4 \pi k^{3} P_{\|}^{s}(k)$.

We use the same definition (46), even though $P_{\|}^{s}(k)$ only holds along the longitudinal direction and $P_{\perp}^{s}(k)(=P(k))$ holds along the two transverse directions (so that using a factor $k$ instead of $k^{3}$ would be more natural here), to make the comparison with Fig. 1 easier. In particular, this means that $\Delta_{\perp}^{2 s}(k)$ is given by Fig. 1 for transverse wavenumbers.

As compared with Fig. 1, we can check that the linear-regime power, $P_{\|}^{s(1)}$, is amplified by a factor $(1+f)^{2}$, whereas standard higher order terms are amplified by factors $(1+f)^{2 n}$, see Eq. (62). Even though the same factors apply to the higher order terms of the "renormalized" perturbative expansion, see Eq. (64), their peak height is not greater than in Fig. 1 for $n \geq 2$ because of the stronger Gaussian damping prefactor $\mathrm{e}^{-k^{2}(1+f)^{2} \sigma_{v}^{2}}$ in Eq. (63). For large $n$ this even leads to a smaller amplitude as compared with the associated real-space contribution. This makes the full nonlinear Zeldovich and "sticky model" power spectra greater than their real-space counterparts on large scales, in the quasi-linear regime, but smaller in the highly nonlinear regime. In particular, it is clear from Eq. (60) that the high- $k$ damping of the Zeldovich power spectrum becomes sharper because of the factor $(1+f)^{2}$ in the exponential term (even though this only leads to a powerlaw decay as noticed in Sect. 3.1). As well as for the real-space 

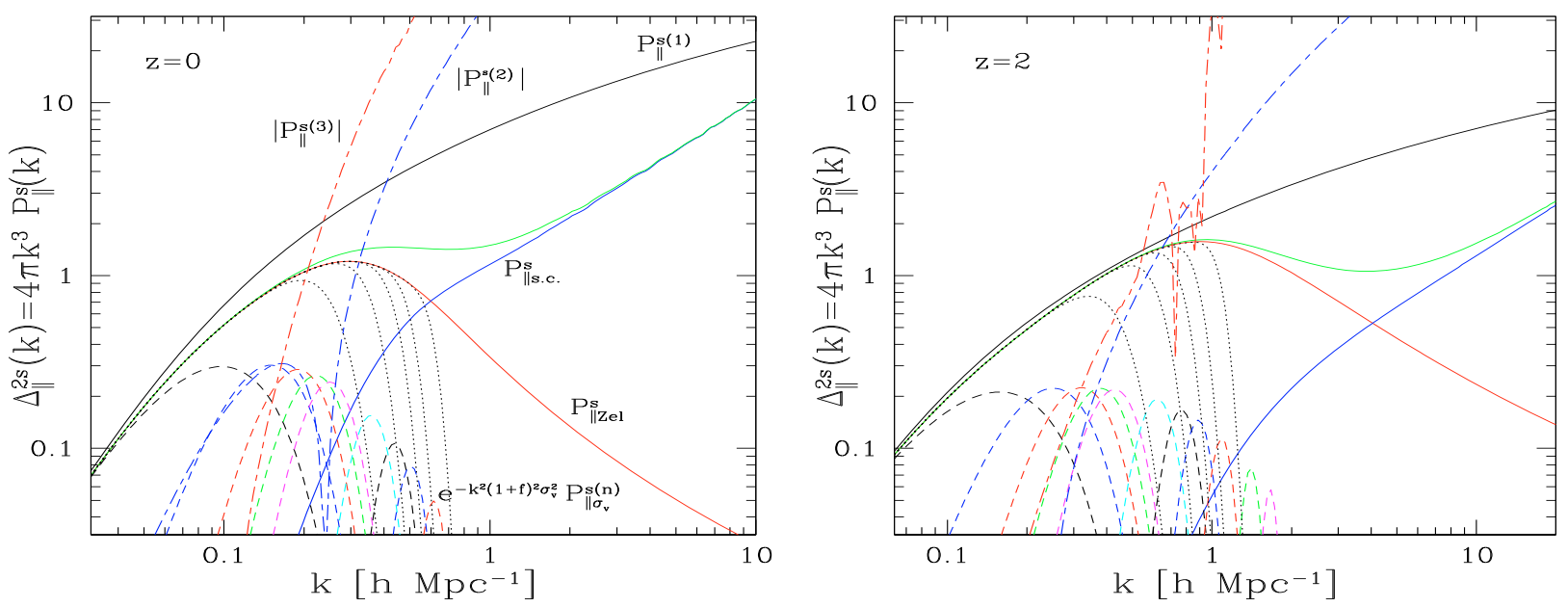

Fig. 4. The logarithmic redshift-space power spectrum, $\Delta_{\|}^{2 s}(k)=4 \pi k^{3} P_{\|}^{s}(k)$, for wavenumbers along the line of sight. Both plots, at $z=0(l e f t$ panel) and $z=2$ (right panel), are the redshift-space counterparts of those shown in Fig. 1 for the real-space power spectrum.

power spectrum, the "renormalized" perturbative expansion (63) is more convenient to distinguish the relative contributions of higher order terms, as they are all positive and do not show the cancellations associated with the standard expansion (62).

Since within the "sticky model" we have after shell crossing $\Delta s_{1}=0$, just as we had $\Delta x_{1}=0$ in real space, we again obtain a $k^{-2}$ tail at high $k$ for the nonperturbative term $P_{\| \text {s.c. }}^{s}(k)$, whence $\Delta_{\| \text {sticky }}^{2 s}(k) \sim k$, as would be the case for planar structures in redshift space. However, because the dominant effect of redshift distortions on small scales is to decrease the power, through damping factors of the form $\mathrm{e}^{-k^{2}(1+f)^{2} \sigma_{v}^{2}}$, the nonperturbative redshift-space contribution $P_{\| \text {s.c. }}^{s}(k)$ is less than its realspace counterpart. Coupled with the faster decay of $P_{\| \mathrm{Zel}}^{s}(k)$, this leads to a temporary decrease of $\Delta_{\| \text {sticky }}^{2 s}(k)$ at $k \sim 1$ to $4 h \mathrm{Mpc}^{-1}$ at $z=2$, before the asymptotic tail $\propto k$ becomes dominant.

The "sticky model" is not intended here to describe the redshift-space power spectrum better than the Zeldovich approximation. Indeed, on small scales setting $\Delta v_{1}=0$ is not realistic, since one should rather describe multi-streaming and take into account the finite velocity dispersion of collapsed objects built by virialization processes. Then, instead of a "sticking" in redshift space one obtains a finite size extension that is greater than the real-space size of the object (because of the additional term associated with the velocity dispersion in Eq. (49)). As is known, this explains the characteristic "fingers of god" observed in galaxy surveys (Jackson 1972; Tadros et al. 1999), and instead of a universal growing tail $\Delta_{\| \text {sticky }}^{2 s}(k) \propto k$, this leads to a decaying tail (as for the Zeldovich power spectrum but with different quantitative properties, since the latter are set by the complex nonlinear virialization processes rather than the linear velocity field). Of course, it is possible to modify the "sticky model" to implement such a velocity dispersion and to obtain more realistic results in redshift space. For instance, while retaining "sticking" in real space, one could allocate a random relative velocity to the pair, so that the perturbative expansions remain unchanged and the nonperturbative contribution involves a damping term of the form $\mathrm{e}^{-k^{2} \sigma_{\text {vir }}^{2}}$, where $\sigma_{\text {vir }}$ is the characteristic velocity dispersion in virialized objects. However, this goes beyond the scope of this article, since here we are only interested in the comparison of perturbative and nonperturbative terms and the simple "sticky model" in the form (66) is sufficient to estimate the scale where nonperturbative corrections become important.

\subsection{Redshift-space perturbative and nonperturbative contributions}

Finally, as for the real-space Fig. 2, we show in Fig. 5 the ratios of the successive terms of the "renormalized" perturbative expansion (63) with respect to the nonlinear power spectrum of the "sticky model", for the same orders $n=1$ to 5, as well as $n=10,15,20,30,50$, and 70 . We also plot the ratio $P_{\| \text {s.c. }}^{s}(k) / P_{\| \text {|sticky }}(k)$, to compare with the amplitude of the nonperturbative correction associated with shell crossing effects, and we consider the four redshifts $z=0,1,2$, and 3 . As for the realspace power spectrum, the potential of perturbative expansions is greater at higher redshift, as we can include more perturbative terms before they become subdominant as compared with the nonperturbative contribution $P_{\| \text {s.c. }}^{s}(k)$, and they extend by a larger factor the range of $k$ where systematic results can be obtained.

We give in Table 2 the wavenumbers $k_{\| 1 \text { loop }}^{s}$ and $k_{\| \text {s.c. }}^{s}$ where the second order of perturbation theory, $P_{\| \sigma_{v}}^{s(2)}(k)$, and the nonperturbative contribution, $P_{\| \text {s.c. }}^{s}(k)$, reach levels of $1 \%, 10 \%$, or $50 \%$. As noticed above from the comparison of Figs. 5 and 2, the interval $\left[k_{\| 1 \text { loop }}^{s}, k_{\| \text {s.c. }}^{s}\right]$, where higher orders of perturbation theory apply, shifts to higher $k$ and becomes broader at higher redshift, as for the real-space spectrum described in Table 1. However, it is shifted towards smaller $k$ than with the real space power spectrum, as could be expected from the fact that redshiftspace perturbative terms of order $n$ are multiplied by prefactors $(1+f)^{2 n}$, see Eqs. $(62)-(64)$, so that higher order terms become relevant earlier and on larger scales. On the other hand, the order $n_{\| \text {s.c. }}^{s}$, after which perturbative contributions become subdominant as compared with the nonperturbative contribution, is similar to the one obtained for the real-space power spectrum, and grows from 8 at $z=0$ up to 72 at $z=3$.

Since redshift distortions are greatest along the line of sight and they vanish for transverse wavenumbers, one would obtain similar results for multipoles of the redshift-space power spectrum, where one expands the dependence on the angle with respect to the line of sight of $P^{s}(\boldsymbol{k})$ over Legendre polynomials of 

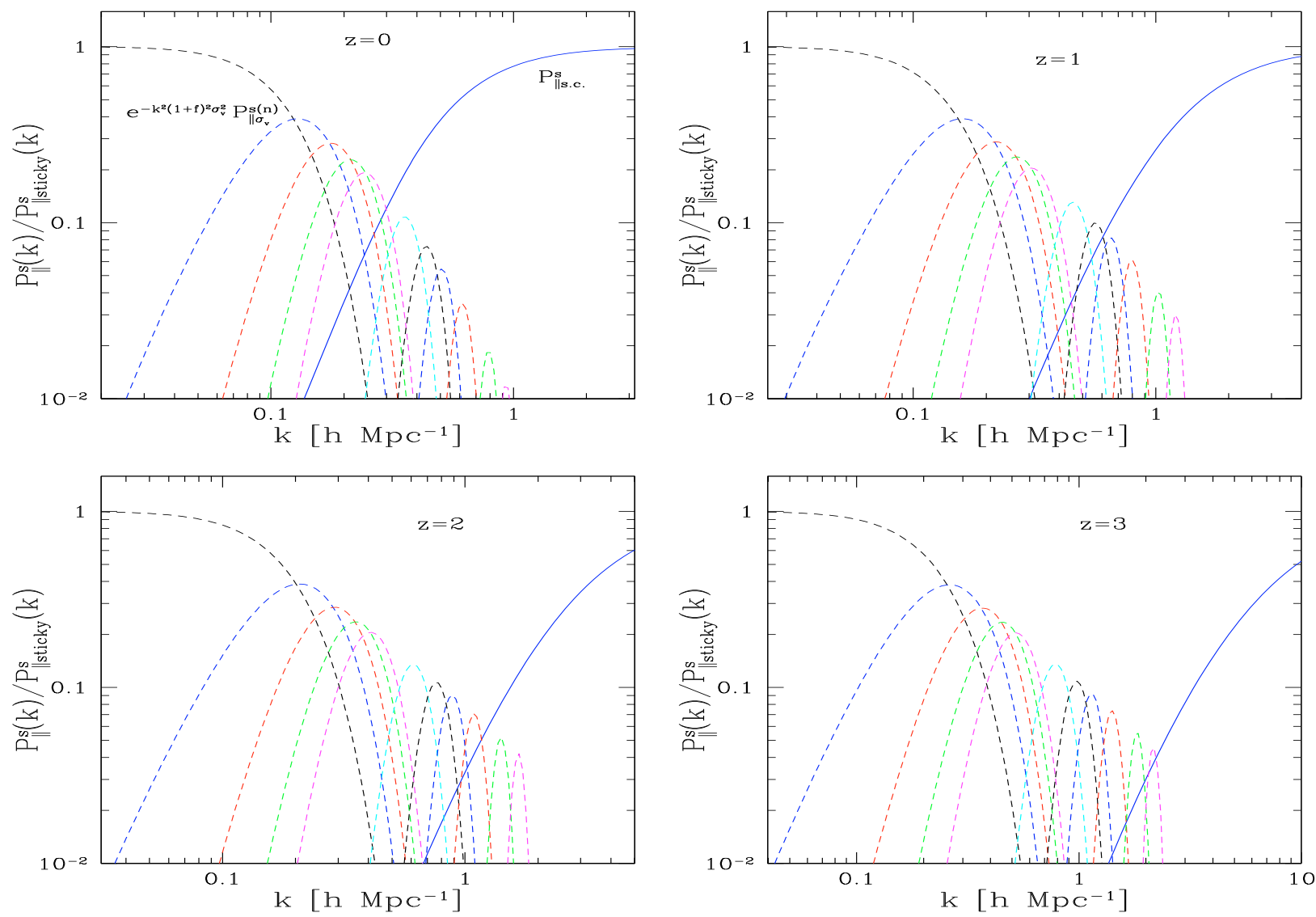

Fig. 5. The ratios of the "renormalized" perturbative terms of the expansion (63) to the nonlinear "sticky model" redshift-space power spectrum, and of the nonperturbative contribution $P_{\| \text {s.c. }}^{s}(k)$. This is the redshift-space counterpart of the real-space Fig. 2, for longitudinal wavenumbers.

Table 2. The dependence on redshift $z$ of the wavenumbers where the ratios $\mathrm{e}^{-k^{2}(1+f)^{2} \sigma_{v}^{2}} P_{\| \sigma_{v}}^{s(2)}(k) / P_{\| \text {sticky }}^{s}(k)$ (at $\left.k_{\| 1 \text { loop }}^{s}\right)$ and $P_{\| \text {s.c. }}^{s}(k) / P_{\| \text {sticky }}^{s}(k)$ (at $k_{\| \text {s.c. }}^{s}$ ) reach $1 \%, 10 \%$, and $50 \%$.

\begin{tabular}{ccccc}
\hline \hline$z$ & $\%$ & $k_{\| 1 \text { loop }}^{s}\left[h \mathrm{Mpc}^{-1}\right]$ & $k_{\| \text {s... }}^{s}\left[h \mathrm{Mpc}^{-1}\right]$ & $n_{\| \text {|s.c. }}^{s}$ \\
\hline \multirow{2}{*}{0} & $1 \%$ & 0.025 & 0.13 & \\
& $10 \%$ & 0.052 & 0.26 & 8 \\
& $50 \%$ & & 0.57 & \\
\hline & $1 \%$ & 0.03 & 0.3 & \\
1 & $10 \%$ & 0.064 & 0.63 & 18 \\
& $50 \%$ & & 1.3 & \\
\hline \multirow{2}{*}{2} & $1 \%$ & 0.033 & 0.68 & \\
& $10 \%$ & 0.08 & 1.5 & 37 \\
& $50 \%$ & & 4 & \\
\hline & $1 \%$ & 0.043 & 1.3 & \\
3 & $10 \%$ & 0.1 & 3.2 & 72 \\
& $50 \%$ & & 9.8 & \\
\hline
\end{tabular}

Notes. The last column gives the last order $n_{\| \text {s.c. }}^{s}$ of the "renormalized" perturbative expansion that is not fully below the nonperturbative term. This table is the redshift-space counterpart of the real-space Table 1, for longitudinal wavenumbers.

$\mu=\left(\boldsymbol{k} \cdot \boldsymbol{e}_{z}\right) / k$. On a quantitative level, one would obtain results in-between those presented in Tables 1 and 2.

\section{Conclusion}

In this article we have introduced a "sticky model" that coincides with the usual Zeldovich dynamics before shell crossing, while after shell crossing it includes a sticking of particle pairs along their longitudinal direction. This implies that the nonlinear density power spectra of both models have the same perturbative expansions and only differ by nonperturbative terms that arise from the dynamics beyond shell crossing. Since we can obtain explicit expressions for perturbative terms at all orders and for this nonperturbative correction, we have been able to compare their respective amplitudes in detail, focusing on a $\Lambda \mathrm{CDM}$ cosmology.

In real space, we find that perturbative approaches based on the fluid description can extend the wavenumber up to which systematic analytical predictions can be obtained from $k \simeq$ $0.033 h \mathrm{Mpc}^{-1}$ up to $\simeq 0.23 h \mathrm{Mpc}^{-1}$ at $z=0$, and from $\simeq 0.07 h \mathrm{Mpc}^{-1}$ up to $\simeq 2.2 h \mathrm{Mpc}^{-1}$ at $z=3$, as compared with linear theory (if we need an accuracy of $1 \%$ ). We also give detailed results for other redshifts and for $10 \%$ and $50 \%$ accuracy levels. Going to higher $k$ requires taking shell-crossing effects into account. Nevertheless, these results show that such perturbative approaches, based on hydrodynamical equations of motion, have a significant potential, especially at high and moderate redshifts. Since it is unlikely that it will be possible to explicitly compute high-order perturbative terms up to the last order $n_{\text {s.c. }}$ which is above the nonperturbative correction $\left(n_{\text {s.c. }}=9\right.$ at $z=0$ and $n_{\text {s.c. }}=66$ at $z=3$ ), this provides a strong incentive to develop resummation schemes. Then, the hope is that such methods can efficiently resum most of the contributions associated with higher order terms and achieve a faster convergence.

This analysis is also useful in the context of cosmological reconstruction, where one tries to recover the dynamical history of a given region of the sky from its present density field 
(and next to derive from this reconstruction the present velocity field). Indeed, these methods usually neglect shell-crossing effects. In particular, we find that the Monge-Ampère-Kantorovich method is close to optimal at $z=0$, as it fares well down to the scale where shell-crossing contributions dominate. With respect to the reconstruction of the baryon acoustic peak or of the acoustic oscillations of the power spectrum, there seems to be only modest room for improvement over current methods based on the linear displacement field at $z=0$, in agreement with only a few orders of perturbation theory being relevant $\left(n_{\text {s.c. }}=9\right)$. At higher $z$ one could in principle do much better by including higher order terms, but since baryon acoustic oscillations of the linear power spectrum have a small amplitude at high $k$, this may not significantly improve the signal-to-noise ratio. However, this remains useful for other observational probes that are sensitive to the shape and amplitude of the power spectrum on weakly nonlinear scales, such as weak-lensing surveys.

We have also pointed out that the behavior of the system after shell crossing plays a key role in the shape of the density power spectrum on mildly nonlinear scales, $1<\Delta^{2}(k)<100$. Thus, arguments based on the spherical collapse dynamics are not sufficient to explain the steep rise of the nonlinear power in this intermediate regime. Indeed, while for the Zeldovich dynamics such a growth is almost entirely wiped out by the escape of particles to infinity (as the random linear displacements erase smallscale features and lead to a decay of $\Delta^{2}(k)$ at high $k$ ), for the "sticky model" there is a regular growth up to the high- $k$ asymptote, $P_{\text {sticky }}(k) \sim k^{-2}$. Therefore, it is not sufficient to consider the spherical collapse of a "typical" overdensity to explain the shape of $P(k)$ on these scales, as one must take the Gaussian average over a whole range of initial density fluctuations, which show a significant amount of shell crossing that has a strong impact on the resulting power spectrum.

Finally, we have obtained similar results for the redshiftspace power spectrum. Again, the scope of perturbative approaches (based on the fluid description) is greater at higher $z$. As compared with the real-space power spectrum, the characteristic wavenumbers where higher order terms of the perturbative expansions and nonperturbative contributions come into play are shifted to lower values of $k$. Indeed, because of the amplification of perturbations from the uniform Hubble background by the additional contribution from peculiar velocities, the nonlinear regime is reached on larger scales than in real space.

The "sticky model" presented in this article, and more generally the expression (7) of the density power spectrum in terms of the Lagrangian displacement, could serve as a basis for models for the nonlinear power spectrum. In particular, the spirit of this model could be incorporated into the Lagrangian-based models, associated with the parametric system (47), (48), or into the "halo model", to make the bridge between the perturbative regime and the highly nonlinear, nonperturbative regime (Valageas \& Nishimichi 2010). This could also be extended to redshift space by including a model for the velocity dispersion within virialized objects. However, this is beyond the scope of the present article so we leave it to future works.

\section{References}

Abramowitz, M., \& Stegun, I. A. 1970, Handbook of Mathematical Functions (New York: Dover)
Afshordi, N. 2007, Phys. Rev. D, 75, 021302

Alcock, C., \& Paczynski, B. 1979, Nature, 281, 358

Bec, J., \& Khanin, K. 2007, Phys. Rep., 447, 1

Bernardeau, F., \& Kofman, L. 1995, ApJ, 443, 479

Bernardeau, F., \& Valageas, P. 2008, Phys. Rev. D, 78, 083503

Bernardeau, F., \& Valageas, P. 2010a, Phys. Rev. D, 81, 043516

Bernardeau, F., \& Valageas, P. 2010b, Phys. Rev. E, 82, 016311

Bernardeau, F., Colombi, S., Gaztañaga, E., \& Scoccimarro, R. 2002, Phys. Rep., 367,1

Bond, J. R., Cole, S., Efstathiou, G., \& Kaiser, N. 1991, ApJ, 379, 440

Brenier, Y., Frisch, U., Henon, M., et al. 2003, MNRAS, 346, 501

Buchert, T. 1994, MNRAS, 267, 811

Carlson, J., White, M., \& Padmanabhan, N. 2009, Phys. Rev. D, 80, 043531

Coles, P., Melott, A. L., \& Shandarin, S. F. 1993, MNRAS, 260, 765

Cooray, A., \& Sheth, R. 2002, Phys. Rep., 372, 1

Crocce, M., \& Scoccimarro, R. 2006a, Phys. Rev. D, 73, 063520

Crocce, M., \& Scoccimarro, R. 2006b, Phys. Rev. D, 73, 063519

Crocce, M., \& Scoccimarro, R. 2008, Phys. Rev. D, 77, 023533

Eisenstein, D. J., Hu, W., \& Tegmark, M. 1998, ApJ, 504, L57

Eisenstein, D. J., Zehavi, I., Hogg, D. W., et al. 2005, ApJ, 633, 560

Eisenstein, D. J., Seo, H.-J., Sirko, E., \& Spergel, D. N. 2007, ApJ, 664, 675

Goroff, M. H., Grinstein, B., Rey, S.-J., \& Wise, M. B. 1986, ApJ, 311, 6

Gurbatov, S. N., Saichev, A. I., \& Shandarin, S. F. 1989, MNRAS, 236, 385

Gurbatov, S., Malakhov, A., \& Saichev, A. 1991, Nonlinear random waves and turbulence in nondispersive media: waves, rays and particles (Manchester University Press)

Hamilton, A. J. S. 1992, ApJ, 385, L5

Hamilton, A. J. S., Kumar, P., Lu, E., \& Matthews, A. 1991, ApJ, 374, L1

Jackson, J. C. 1972, MNRAS, 156, 1P

Kaiser, N. 1987, MNRAS, 227, 1

Kraichnan, R. H. 1959, J. Fluid Mech., 5, 497

Massey, R., Rhodes, J., Leauthaud, A., et al. 2007, ApJS, 172, 239

Matarrese, S., \& Pietroni, M. 2007, JCAP, 6, 26

Matsubara, T. 2008, Phys. Rev. D, 77, 063530

McDonald, P. 2007, Phys. Rev. D, 75, 043514

Melott, A. L., Shandarin, S. F., \& Weinberg, D. H. 1994, ApJ, 428, 28

Mohayaee, R., Mathis, H., Colombi, S., \& Silk, J. 2006, MNRAS, 365, 939

Munshi, D., Valageas, P., van Waerbeke, L., \& Heavens, A. 2008, Phys. Rep., 462,67

Navarro, J. F., Frenk, C. S., \& White, S. D. M. 1997, ApJ, 490, 493

Noullez, A., \& Vergassola, M. 1994, J. Sci. Comput., 9, 259

Padmanabhan, T. 1996, MNRAS, 278, L29

Pauls, J. L., \& Melott, A. L. 1995, MNRAS, 274, 99

Peacock, J. A., \& Dodds, S. J. 1996, MNRAS, 280, L19

Peebles, P. J. E. 1974, A\&A, 32, 391

Peebles, P. J. E. 1980, The large scale structure of the universe (Princeton: Princeton university press)

Peebles, P. J. E. 1982, ApJ, 263, L1

Peebles, P. J. E. 1989, ApJ, 344, L53

Pietroni, M. 2008, JCAP, 10, 36

Sathyaprakash, B. S., Sahni, V., Munshi, D., Pogosyan, D., \& Melott, A. L. 1995, MNRAS, 275, 463

Schneider, P., \& Bartelmann, M. 1995, MNRAS, 273, 475

Seo, H.-J., Eckel, J., Eisenstein, D. J., et al. 2010, ApJ, 720, 1650

Smith, R. E., Peacock, J. A., Jenkins, A., et al. 2003, MNRAS, 341, 1311

Tadros, H., Ballinger, W. E., Taylor, A. N., et al. 1999, MNRAS, 305, 527

Taruya, A., \& Hiramatsu, T. 2008, ApJ, 674, 617

Taruya, A., Nishimichi, T., Saito, S., \& Hiramatsu, T. 2009, Phys. Rev. D, 80, 123503

Taylor, A. N., \& Hamilton, A. J. S. 1996, MNRAS, 282, 767

Valageas, P. 2004, A\&A, 421, 23

Valageas, P. 2007a, A\&A, 465, 725

Valageas, P. 2007b, A\&A, 476, 31

Valageas, P. 2008, A\&A, 484, 79

Valageas, P. 2009, Phys. Rev. E, 80, 016305

Valageas, P., \& Schaeffer, R. 1997, A\&A, 328, 435

Valageas, P., \& Bernardeau, F. 2010, Phys. Rev. D, submitted [arXiv: 1009. 1974]

Valageas, P., \& Nishimichi, T. 2010, A\&A, submitted [arXiv: 1009. 0597]

Vergassola, M., Dubrulle, B., Frisch, U., \& Noullez, A. 1994, A\&A, 289, 325

Weinberg, D. H., \& Gunn, J. E. 1990, MNRAS, 247, 260

Zeldovich, Y. B. 1970, A\&A, 5, 84 